\title{
1 Deamidated TPI is an efficacious target for cell-selective therapy in triple-negative \\ 2 breast cancer
}

4 Sergio Enríquez-Flores, ${ }^{1, *}$ Luis A. Flores- López, ${ }^{1,2}$ Ignacio De la Mora-De la Mora, ${ }^{1}$ Itzhel 5 García-Torres, ${ }^{1}$ Isabel Gracia-Mora, ${ }^{3}$ Pedro Gutiérrez-Castrellón, ${ }^{4}$ Cynthia Fernández6 Lainez, ${ }^{5,7}$ Yoalli Martínez-Pérez, ${ }^{1}$ Alberto Olaya-Vargas, ${ }^{6}$ Paul de Vos, ${ }^{7}$ Gabriel López7 Velázquez, ${ }^{1, *}$

9 ' ${ }^{1}$ Laboratorio de Biomoléculas y Salud Infantil, Instituto Nacional de Pediatría, CDMX, 10 México

$11{ }^{2}$ CONACYT Instituto Nacional de Pediatría, CDMX, México

$12{ }^{3}$ Directora de la Unidad de Investigación Preclínica, Facultad de Química, Universidad 13 Nacional Autónoma de México, CDMX, México

$14{ }^{4}$ Hospital General Dr. Manuel Gea González, México;

$15{ }^{5}$ Laboratorio de Errores Innatos del Metabolismo y Tamiz, Instituto Nacional de Pediatría, 16 CDMX, México

$17{ }^{6}$ Stem Cell Transplantation and Cellular Therapy, Instituto Nacional de Pediatría, CDMX, 18 México

$19{ }^{7}$ Department of Pathology and Medical Biology, University of Groningen, University 20 Medical Center Groningen, Groningen, 9713 GZ, the Netherlands

$21{ }^{8}$ Posgrado en Ciencias Biológicas, Universidad Nacional Autónoma de México, CDMX, 22 México

23 *Correspondence: glv_1999@ciencias.unam.mx, sergioenriquez@ciencias.unam.mx

24 Key words: cancer/drug repurposing/drug target/glycolysis/methyl glyoxal/triosephosphate 25 isomerase. 


\section{Abstract}

33 Human TPI (HsTPI) is a central and essential glycolytic enzyme for energy supply and is

34 overexpressed in cancer cells. Here, we investigated HsTPI as a potential target for

35 inducing cell death in triple-hormone receptor-negative breast cancer, which is highly

36 dependent on glycolysis, and therapies for its treatment are limited. We found endogenous

37 accumulation of deamidated HsTPI in human breast cancer cells, which might be caused by

38 the lower activity of the HsTPI-degrading caspase-1 in breast cancer cells. In silico and in

39 vitro analyses of deamidated HsTPI demonstrated the efficacy of thiol-reactive drugs in

40 blocking enzyme activity. The cancer cells were selectively programmed to undergo

41 apoptosis with thiol-reactive drugs by inducing the production of methylglyoxal (MGO)

42 and advanced glycation-end products (AGEs). In vivo in mice, the thiol-reactive drug

43 effectively inhibited the growth of human tumors by targeting HsTPI as underlying

44 mechanism. Our findings demonstrate deamidated HsTPI as a novel target to develop

45 therapeutic strategies for treating cancers and other pathologies in which this post-

46 translationally modified protein accumulates. 
63 Since cancer cells show a high capacity for proliferation, they are energy demanding which

64 is driven by glycolysis[1-3]. Due to this high glycolysis and upregulation of glycolysis-

65 associated enzymes in cancer cells[4-6], substrate analogs for glycolytic enzymes have

66 been proposed as potential anticarcinogenic therapeutics[7-9] Triosephosphate isomerase

67 (TPI or TIM) is such a potential target for these drugs as it plays a central role in the energy

68 generating phase of glycolysis by isomerizing dihydroxyacetone phosphate (DHAP) to D-

69 glyceraldehyde-3-phosphate (GAP). Regions distinct from catalytic sites are promising for

70 efficacious anti-tumor strategies. These arguments were the rational to investigate the use

71 of deamidated human TPI (HsTPI) as a druggable target[10].

72 In eukaryotes, deamidation is a spontaneous modification of proteins that has repercussions

73 for the proteins' activity and stability, both in vivo and in vitro[11-13]. Especially,

74 asparagine (Asn) and glutamine (Gln) are targets for deamidation in tumor cells.

75 Asparagine deamidation into aspartic acid (Asp) and isoAsp results in alteration of the

76 primary structure of the protein, introducing a negative charge, and often modifying their

77 tertiary structure. As a consequence, functional aspects of the protein are lost interfering

78 with stability of the cells.

79 Two deamidation sites in HsTPI at positions 16 and 72 have been found. Deamidation of

80 Asn 16 is the most common and induces major functional and structural alterations of

81 HsTPI [12]. Continuous catalytic cycles promote the deamidation of HsTPI [14], which is

82 boosted in aged cells as well as in cancer cells [15]. Studies on the structural characteristics

83 of deamidated HsTPI have demonstrated that it is prone to selective inhibition with thiol-

84 reactive compounds [10] and therefore be an effective approach to target HsTPI and there

85 with interfering with glycolysis in cancer cells.

86 Since glycolysis is not adequately regulated in cancer cells, we hypothesized that

87 deamidated HsTPI commonly accumulates in these cells, enabling its use as an efficient

88 druggable target to selectively exert cell death. Here, the presence and accumulation of

89 deamidated HsTPI were demonstrated in triple-hormone receptor negative breast cancer

90 cells, which showed high sensitivity to the thiol-reactive drugs rabeprazole and auranofin.

91 Both drugs inhibited cellular HsTPI enzyme activity and induced selective cell death. Nude

92 immunocompromised mice with implanted human breast cancer cells treated with

93 rabeprazole exhibited significantly reduced tumor size compared with the tumor size in 
94 untreated mice. Additionally, pretreatment of cancer cells with rabeprazole did not lead to

95 tumor formation after implantation in mice. The central finding of our study is that

96 deamidated HsTPI is naturally present and accumulates in cancer cells but not in noncancer

97 cells. Overall, the results of in vitro (recombinant protein), in situ (cell culture) and in vivo

98 (xenograft murine model) experiments demonstrate that deamidated HsTPI is an efficient

99 druggable target.

100

101 RESULTS.

102 Deamidated HsTPI is highly inactivated and structurally blocked by the drugs 103 rabeprazole and auranofin through Cys modification.

104 HsTPI is a homodimeric enzyme for which deamidation occurs at amino acid residues

105 located in the contact site between its two subunits (interface), increasing its accessibility

106 for small ligands[10, 12]. To gain more insight in its active ligand binding sites, we

107 performed in silico and in vitro analyses of crystallographic structures of nondeamidated

108 and deamidated HsTPI and the corresponding recombinant proteins. The largest and

109 deepest cavity at the interface was found in deamidated HsTPI. Docking of rabeprazole and

110 auranofin identified more binding sites in deamidated HsTPI than in nondeamidated HsTPI

111 (Fig. 1a).

112 Consistent with the docking data, our functional in vitro studies with human breast cancer

113 cells showed that in contrast to nondeamidated HsTPI, its deamidated counterpart was

114 selectively bound by both drugs, which completely inhibited its enzyme activity (Fig. 1b,

115 right). Additionally, inhibition of deamidated HsTPI was dose dependent, and deamidated

116 HsTPI was 4-fold more sensitive to rabeprazole and 30-fold more sensitive to auranofin

117 than nondeamidated HsTPI.

118 The main mechanism of action of both drugs (rabeprazole and auranofin) is covalent

119 binding to the thiolate moieties of cysteine residues (Cys) (named Cys derivatization) [16,

120 17]. The primary structure of HsTPI contains 5 Cys residues per subunit, which were

121 visualized and quantified in the recombinant enzyme in absence of the drugs. Treatment

122 with either rabeprazole or auranofin derivatized 4 Cys residues per subunit in deamidated

123 HsTPI, whereas nondeamidated HsTPI contained only one derivatized Cys residue per

124 subunit after drug treatment (Fig. 1b, and Suppl. Table 1). 
126 In addition to these functional studies, we carried out spectroscopic studies to identify 127 structural changes due to drug treatment. To that end, we analyzed the intrinsic 128 fluorescence of HsTPI to follow changes in the exposure of its aromatic residues to solvent 129 in presence of the drugs. The intrinsic fluorescence spectra of nondeamidated HsTPI 130 showed marginal changes in the tertiary structure of the enzyme upon treatment with either 131 rabeprazole or auranofin (Fig. 1c, left). In contrast, the intrinsic fluorescence spectra of 132 deamidated HsTPI upon treatment with both drugs decreased by more than 50\% (Fig. 1c, 133 right). Under these conditions, rabeprazole and auranofin generated a $70 \%$ and $56 \%$ 134 decrease in fluorescence intensity and shifts in the maximum fluorescence emission $\left(\lambda_{\max }\right)$ 135 of 12 and $9.5 \mathrm{~nm}$, respectively (Fig. 1c, right). A shift of $\lambda_{\max }$ toward a longer wavelength 136 (redshift) indicates exposure to tryptophan residues previously buried into a folded 137 structure.

138 To substantiate proof for the structural changes of deamidated HsTPI and the drugs, 139 extrinsic fluorescence studies with 8-anilinonaphthalene-1-sulfonic acid (ANS) were 140 performed. ANS is a fluorescent molecular probe that has a high affinity for hydrophobic 141 regions; thus, proteins containing more hydrophobic cavities will exhibit higher ANS 142 fluorescence signals. The ANS fluorescence in the untreated and treated nondeamidated 143 HsTPI showed a marginal signal (Fig. 1d, left). In contrast, the fluorescence signal from 144 ANS in deamidated HsTPI was strongly increased under all conditions. This signal was 145 augmented with respect to that of the untreated enzyme by 6.5 - and 8-fold upon treatment 146 with rabeprazole and auranofin, respectively (Fig. 1d, right). Additionally, the $\lambda_{\max }$ shifted

147 by $14 \mathrm{~nm}$ to a lower wavelength (shift to blue) with both drugs. These results show that as a 148 consequence of the drug treatments, deamidated HsTPI became highly accessible to small 149 molecules such as ANS. 
A

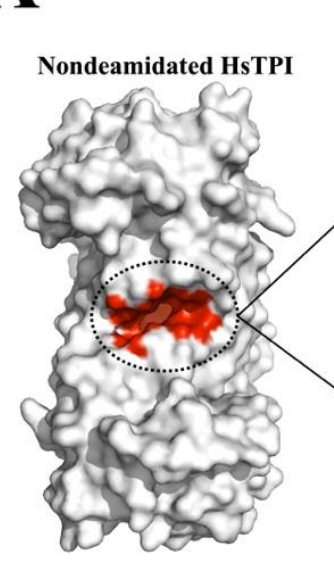

Demanasenturr
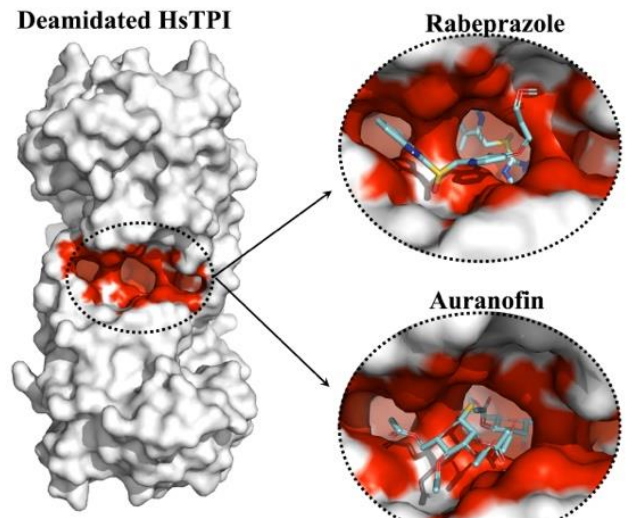

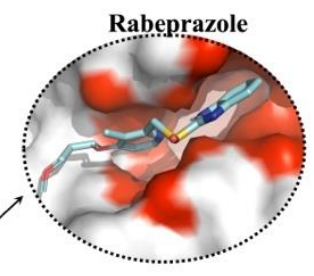

Auranofin
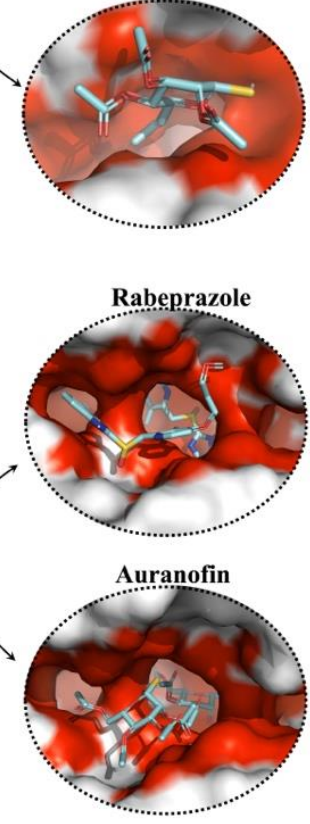

B
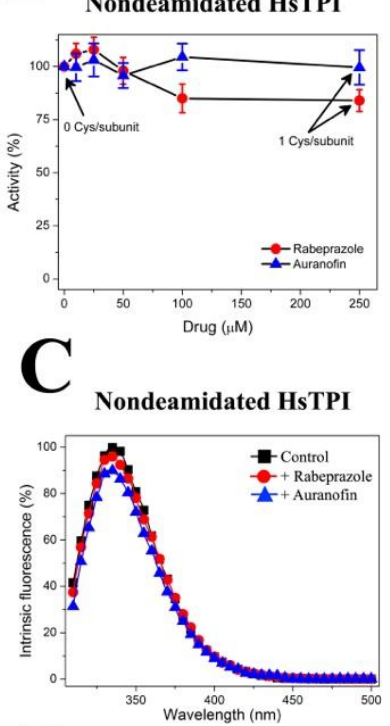

D

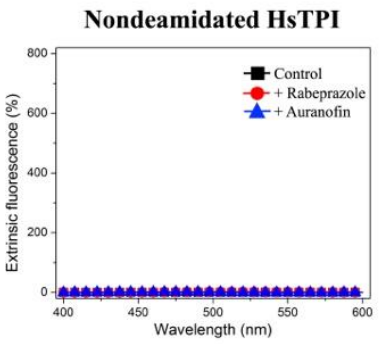

Deamidated HsTPI

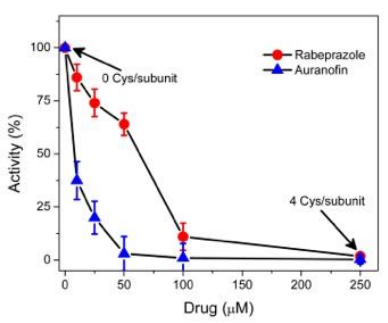

Deamidated HsTPI
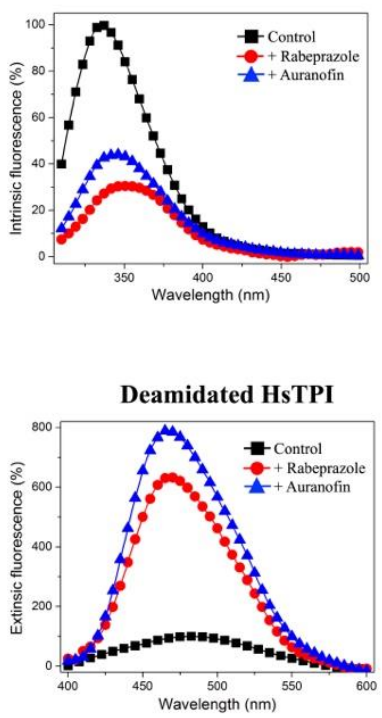

Fig. 1. Deamidated HsTPI was more likely to be functionally and structurally disturbed by the thiolreactive drugs rabeprazole and auranofin than its nondeamidated counterpart. a, Ligand docking in nondeamidated and deamidated enzymes. Rabeprazole and auranofin were docked at the interface of the crystallographic structure. As shown, the deamidated protein incorporates more ligands in the innermost part of the interface than its nondeamidated counterpart. b. Inactivation assays with recombinant nondeamidated and deamidated HsTPI proteins, with rabeprazole $(\bullet)$ or auranofin $(\bullet)$. After incubation, the residual enzymatic activity was determined. The results are expressed as the percent activity, with the enzyme activity without the drug set at $100 \%$. c, Intrinsic fluorescence of nondeamidated and deamidated HsTPI with or without drug treatment, in the absence ( $\bullet$ ) (control) or presence of $250 \mu \mathrm{M}$ rabeprazole $(\bullet)$ or $250 \mu \mathrm{M}$ auranofin $(\Delta)$. The results are expressed as the percent fluorescence, with the maximum fluorescence

162 emission of the control enzyme set at $100 \%$. d, Extrinsic fluorescence of ANS corresponding to the enzymes 163 with or without drug treatment in the absence ( $($ ) (control) or presence of $250 \mu \mathrm{M}$ rabeprazole (•) or $250 \mu \mathrm{M}$ 164 auranofin $(\Delta)$. After incubation, the extrinsic fluorescence was determined by adding $100 \mu \mathrm{M}$ ANS. The 165 background was always subtracted. The results are expressed as percent of fluorescence, with the maximum 166 fluorescence emission of the control in deamidated HsTPI set at 100\%. The results represent the mean of four 
independent experiments.

\section{Deamidated HsTPI is present and accumulates in breast cancer cells.}

170 To study possible endogenous accumulation of deamidated HsTPI in breast cancer cells, a 171 previously validated method of selective cleavage by hydroxylamine was used[18, 19].

172 Hydroxylamine can selectively cleave the intermediary succinimide that is formed by the 173 side chains of Asn and glycine (Gly) in nondeamidated proteins under alkaline conditions; 174 furthermore, in deamidated proteins, a chain composed of Asp-Gly (or isoAsp-Gly) does 175 not form succinimide under the same conditions. Therefore, the deamidated protein is not 176 cleaved[20]. Then, as HsTPI contains two Asn-Gly pairs at positions 16-17 and 72-73, 177 cleavage might generate three peptides that are $1.87,5.96$ and $18.87 \mathrm{kDa}$ in size if the

178 enzyme is not deamidated. When the cleavage of nondeamidated HsTPI is incomplete, a 179 peptide with a size of $7.83 \mathrm{kDa}$ could also be produced. On the other hand, the cleavage of 180 deamidated HsTPI at only position 16 would produce peptides with sizes of 7.83 and 18.87

$181 \mathrm{kDa}$, whereas double deamidated HsTPI would not be cleaved at position 16 or 72 . This 182 difference in cleavage patterns of the recombinant enzymes versus cellular HsTPI from 183 human primary mammary epithelial cells (HMECs) (normal breast cells) and MDA-MB-

184231 cells (triple-negative breast cancer cells) was used to study differences in accumulation 185 of deamidated HsTPI.

186 HsTPI from HMECs showed the same cleavage pattern found for recombinant 187 nondeamidated HsTPI (Fig. 2a, lane 6 vs lane 3), whereas HsTPI from MDA-MB-231 cells 188 showed a cleavage pattern similar to that of recombinant HsTPI deamidated at position 16 189 (Fig. 2a, lane 7 vs lane 4). The cleavage selectivity of the hydroxylamine method was 190 reinforced by the absence of a cleavage pattern for the recombinant double deamidated 191 HsTPI at positions 16 and 72 (Fig. 2a, lane 5). Herewith we demonstrated that deamidated 192 HsTPI was present in these cancer cells and not in their noncancerous counterparts.

193 Since every deamidation reaction adds one negative charge to HsTPI, native 194 polyacrylamide gel electrophoresis (nPAGE) can be applied for analyzing the acidic status 195 of both recombinant and cellular HsTPI proteins. The electrophoretic mobility patterns 196 resulting from this immunoblotting of the nPAGE gel clearly showed and confirmed that in 197 cancer cells deamidated HsTPI accumulated (Fig. 2b, cancer cells, lane 4), which was not 
198 observed in normal cells (Fig. 2b, normal cells lanes 4). These observations were the main 199 rational to propose and test drugs that target and inhibit deamidated HsTPI to selectively 200 delete cancer cells and keep non-cancerous cells virtually untouched.

$\mathbf{a}$

b
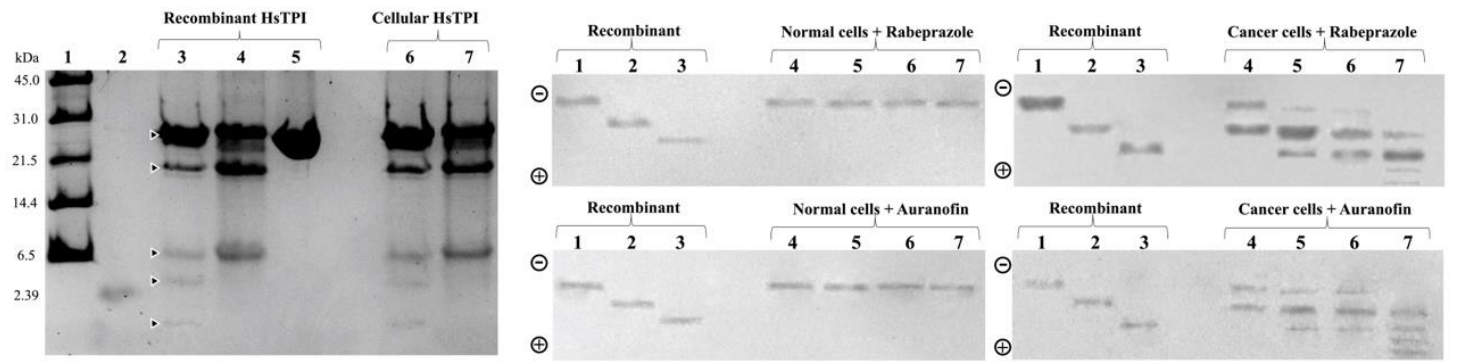

c
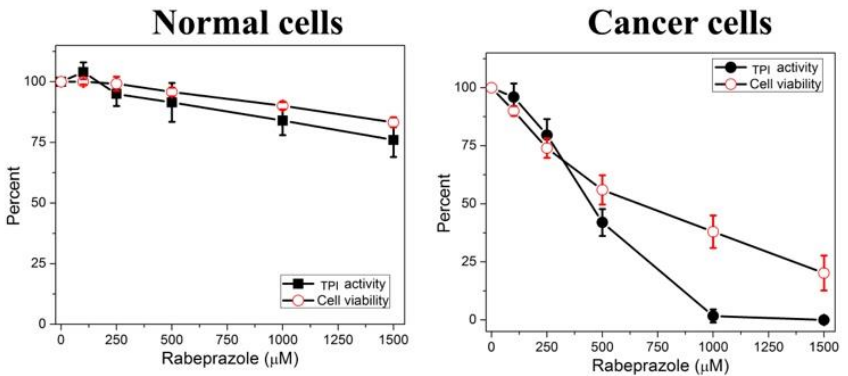

Normal cells
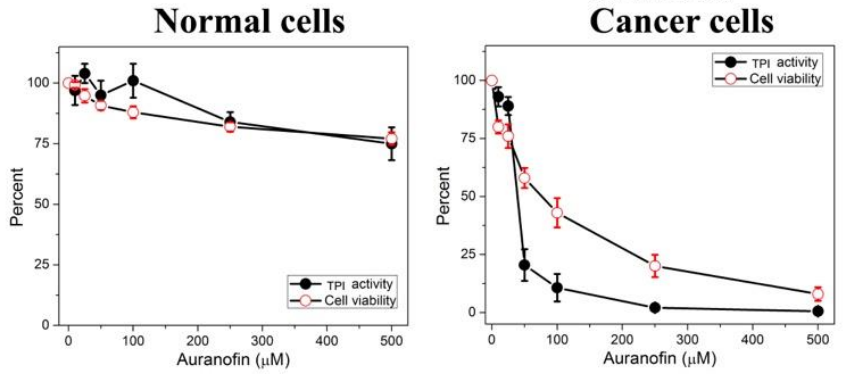

Fig. 2. Deamidated HsTPI accumulated in breast cancer cells was affected by treatment with rabeprazole and auranofin. a, Hydroxylamine cleavage of the recombinant and cellular HsTPI proteins. Lane 1: molecular weight standard (broad range, Bio-Rad), lane 2: peptide with a size of $2.39 \mathrm{kDa}$, lanes 3 to 5: (10 $\mu \mathrm{g} / \mathrm{lane})$ nondeamidated (WT), once deamidated (N16D) and double deamidated (N16D/N72D) HsTPI proteins. Lanes 6 and 7: (20 $\mu \mathrm{g} / \mathrm{lane})$ immunoprecipitated HsTPI from HMECs and MDA-MB-231 cells, respectively. The cleavage pattern of the nondeamidated recombinant enzyme (lane 3) is similar to that of cellular HsTPI from normal cells (HMECs) (lane 6). In the same manner, the cleavage pattern of the deamidated recombinant enzyme (lane 4) is similar to that of cellular HsTPI from cancer cells (MDA-MB-

211 231) (lane 7). b, Western blot analysis of recombinant and cellular HsTPI in nPAGE. In the four panels, lanes

2121 to 3 (10 $\mu \mathrm{g}$ of protein/lane) correspond to the recombinant nondeamidated, once deamidated and double 213 deamidated HsTPI, respectively. Lanes 4 to 7 (100 $\mu \mathrm{g}$ protein/lane) correspond to proteins from the protein 214 extract from normal cells (HMECs) (left panel, upper and bottom) and cancer cells (MDA-MB-231) (right 
215 panel, upper and bottom) treated with $0,500,1000$ and $1500 \mu \mathrm{M}$ rabeprazole (upper) or 0, 25, 50 and $100 \mu \mathrm{M}$

216 auranofin (bottom). c, HsTPI enzyme activity and viability of HMECs and MDA-MB-231 cells treated with

217 rabeprazole and auranofin. The results are expressed as percentages of residual enzyme activity, with the

218 values obtained in the absence of the drugs set at $100 \%$. The results are the mean of four independent

219 experiments.

221 Rabeprazole and auranofin induce the formation of inactive acidic isoforms of HsTPI 222 in cancer cells and promoted selective cell death

223 Negatively charged HsTPI isoforms (acidic isoforms) appeared after the treatment of 224 cancer cells (Fig. 2b, cancer cells, lanes 5-7) but not in normal cells (i.e. noncancer cells) 225 (Fig. 2b, lanes 5-7) with rabeprazole or auranofin. Furthermore, the naturally accumulated 226 deamidated HsTPI already present in the cancer cells tended to disappear, and other more 227 negatively charged isoforms appeared as a result of treatment with increasing doses of both 228 drugs. Importantly, both drug treatments induced the disappearance of nondeamidated 229 HsTPI, which potentiates the effect of the drugs on cancer cells.

230 Cellular HsTPI in cancer cells completely lost its enzyme activity upon treatment with 1000 $231 \mu \mathrm{M}$ rabeprazole, and the viability of these cells decreased to approximately $80 \%$ at 1500 $232 \mu \mathrm{M}$ rabeprazole treatment (Fig. 2c, cancer cells, rabeprazole). Auranofin strongly reduced 233 cellular HsTPI levels in cancer cells at lower concentration. With $250 \mu \mathrm{M}$ auranofin 234 enzyme activity was totally inhibited and cell viability was reduced to $80 \%$. At a 235 concentration of $500 \mu \mathrm{M}$ auranofin totally eliminated cell viability (Fig. 2c, cancer cells, 236 auranofin). These drugs exerted effects on the enzyme activity of cellular HsTPI similar to 237 those obtained for the recombinant enzymes.

238 In contrast, in normal cells, the two drugs induced no more than a $25 \%$ loss in both enzyme 239 activity and cell viability (Fig. 2c, normal cells, rabeprazole and auranofin) again 240 illustrating its selectivity for cancerous cells.

241 Overall, these results show that HsTPI in cancer can be selectively targeted, supporting the 242 crucial involvement of this protein in maintaining viability in breast cancer cells and the 243 selective cell death that can be induced by the tested drugs.

244 To support the notion that the inhibition of cellular HsTPI was attributable to the drug 245 treatments instead of the consequence of cell damage, assays with high drug concentrations 246 and short incubation times were performed. HsTPI activity in breast cancer cells treated 
247 with rabeprazole was almost totally abolished at $2 \mathrm{~h}$ of treatment, and the viability of the

248 cells was close to $100 \%$ for $4 \mathrm{~h}$ until it dropped by approximately $20 \%$ in the last hour

249 (Suppl. Fig. 1, cancer cells, rabeprazole). With auranofin, HsTPI activity was completely

250 abolished at $4 \mathrm{~h}$, whereas cell viability remained close to $100 \%$ and tended to decrease after

$2515 \mathrm{~h}$ of treatment (Suppl. Fig. 1, cancer cells, auranofin). This effect was selective for

252 cancerous cells as HsTPI activity in normal cells dropped by not more than $30 \%$ with

253 rabeprazole treatment, while auranofin treatment exerted a maximum inhibitory effect of

$25410 \%$. The viability of these cells was maintained close to $100 \%$ during treatment (Suppl.

255 Fig. 1, normal cells).

256 These results again support the observation that TIM in cancer cells is a cell-specific target

257 of both drugs that is inactivated prior to cell death, whereas TIM in normal cells is

258 practically unaffected. To assess the hypothesis that the drugs direct their effect to regions

259 other than the catalytic site, the kinetics of cellular HsTPI were determined for the latter

260 assayed conditions in untreated and treated cells. The $K_{\mathrm{m}}$ values for TIM in untreated and

261 treated cancer cells were similar; however, the $\mathrm{V}_{\max }$ values were 3.3 (with rabeprazole) and

2622.6 (with auranofin) times lower than those in untreated cells (Suppl. Fig. 2 and Suppl.

263 Table 2). Consistent with previous reports[12, 21], the $K_{\mathrm{m}}$ values of TIM in normal cells, 264 either untreated or treated with rabeprazole and auranofin, were similar (Suppl. Fig. 2, and

265 Suppl. Table 2). These results support the idea that these drugs do not compete with the 266 active site of HsTPI and that their mechanism of inactivation is noncompetitive.

268 Targeting deamidated HsTPI decreased lactate production and induced excessive 269 production of methylglyoxal and AGEs in breast cancer cells

270 Targeting HsTPI disturbs the glycolytic pathway, which can be demonstrated and 271 quantifying by measuring lactate production. Breast cancer cells, which normally produce 272 high quantities of lactate, significantly decreased their production of lactate when treated 273 with either rabeprazole or auranofin, whereas normal cells were not affected (Fig. 3a). This 274 supports the idea that, when HsTPI is affected by these drugs, glycolytic flux is depleted in 275 cancer cells (Fig. 3a, cancer cells). Additionally, the malfunctioning of HsTPI causes the 276 accumulation of DHAP, the spontaneous degradation of which leads to methylglyoxal 277 (MGO) formation, which in turn inhibits glycolytic flux [22]. An increase in MGO is 
278 related to HsTPI failure, documented in several human disorders [23]. Concordantly, our

279 results showed that the tested treatments increased the production of MGO, but such

280 production was significantly higher in cancer cells than in normal somatic cells (Fig. 3b,

281 cancer cells).

282 MGO is harmful to several cell types, mainly as it is an essential intermediate in the

283 formation of AGEs. In this regard, both drug treatments significantly increased the levels of

284 AGEs in a dose-dependent manner in cancer cells (Fig. 3c, cancer cells) but not in normal

285 cells, in which these products were present at minimal concentrations (Fig. 3c, normal

286 cells). These results are consistent with those observed for MGO formation upon the

287 treatments. Hence, the behavior of deamidated HsTPI leads to the production of toxic

288 metabolites (i.e., MGO and AGEs), which in turn should be involved in the selective cell

289 death process that we observed.
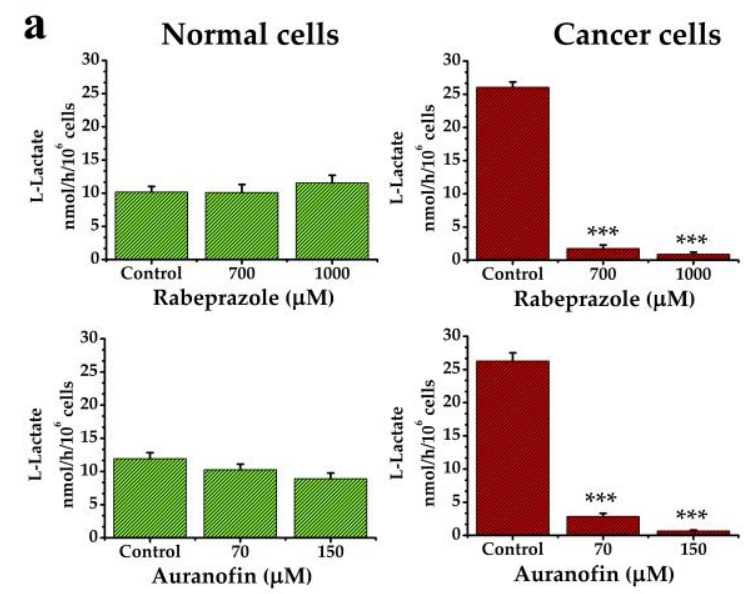

c
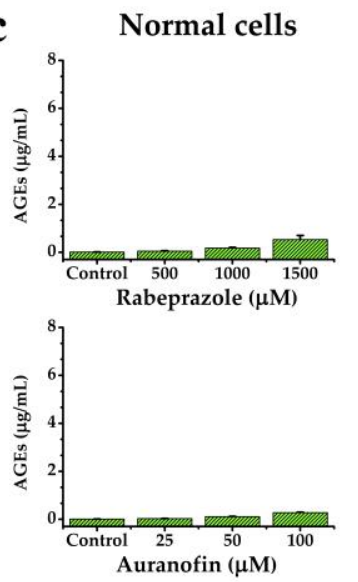
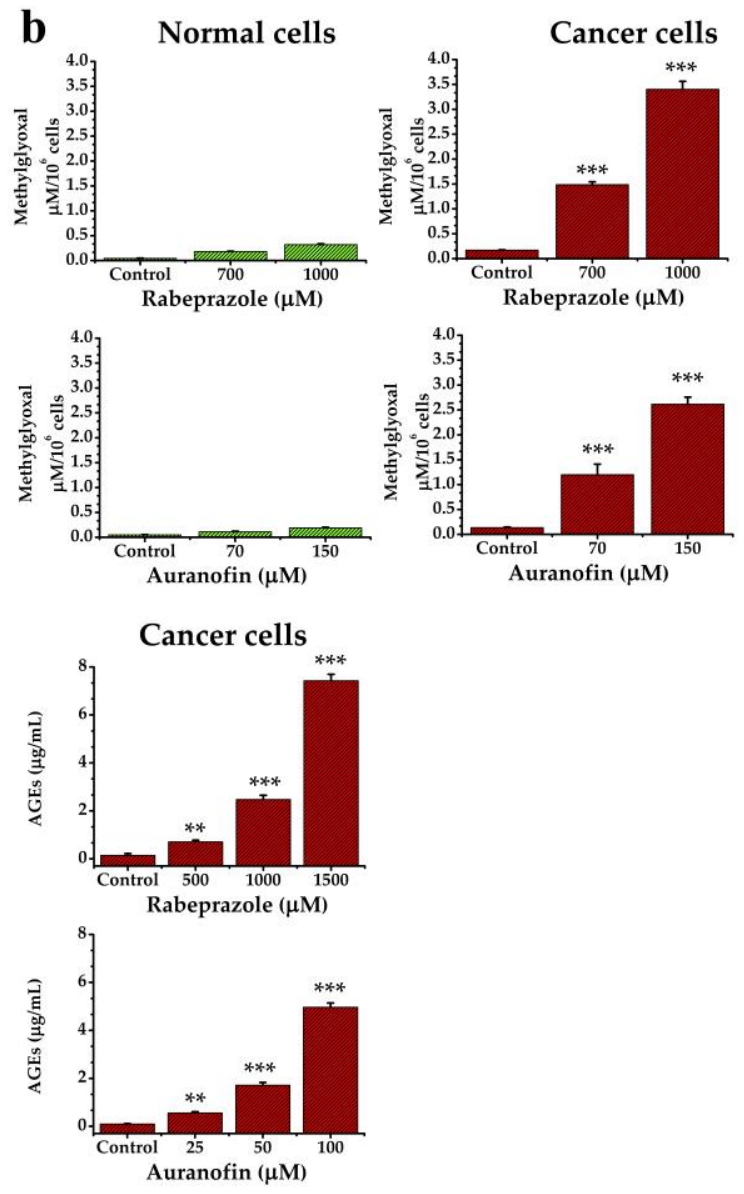
Fig. 3. Quantification of metabolites in normal and cancer cells under drug treatments. a, Determination of extracellular lactate levels in normal and cancer cells. Cells were incubated with 0,700 , and $100 \mu \mathrm{M}$ of rabeprazole or 0,75 , and $150 \mu \mathrm{M}$ of auranofin. The extracellular lactate level is shown. $\mathbf{b}$, Determination of MGO levels in normal and cancer cells with rabeprazole $(0,700$, and $1000 \mu \mathrm{M})$ or auranofin $(0,75$ and 150 $\mu \mathrm{M})$. Intracellular MGO is shown. c, Determination of AGE levels in normal and cancer cells. with 0,500 , 1000 and $1500 \mu \mathrm{M}$ rabeprazole or $0,25,50$ and $100 \mu \mathrm{M}$ auranofin. All results represent the mean of at least four independent experiments. Differences among groups were assessed with one-way ANOVA and Tukey's test with $p$ value $=0.001 * * *$ or with $p$ value $=0.01 * *$

\section{Breast cancer cells treated with rabeprazole and auranofin underwent apoptosis.}

303 Enhancing apoptosis is important to prevent tumor initiation, progression and metastasis 304 formation [24]. As shown in our previous experiments, rabeprazole and auranofin induce 305 overproduction of MGO and AGEs. Both metabolites are known to induce apoptosis [25, 306 26]. To test the influence of rabeprazole and auranofin on cell apoptosis, we analyzed the 307 expression of proteins related to pro- and antiapoptotic events [27] induced through 308 intrinsic pathways (Bax and Bcl-2) [28, 29].

309 The results showed that both drug treatments induced a decrease in the expression of total 310 ERK 1/2 and Bcl-2. Additionally, phosphorylated ERK 1/2 levels were decreased (Fig. 4a).

311 These elements are related to lower cellular proliferation. On the other hand, the 312 proapoptotic elements Bax and cleaved procaspase-7 were increased after both treatments 313 in a dose-dependent manner (Fig. 4a). Additionally, transferase dUTP nick end labeling 314 (TUNEL) assays with breast cancer cells treated with rabeprazole or auranofin showed an 315 increase in apoptotic cells after $24 \mathrm{~h}$ of treatment (Fig. 4b). These results show that our 316 strategy overcomes the antiapoptotic tendencies of cancer cells. 
a
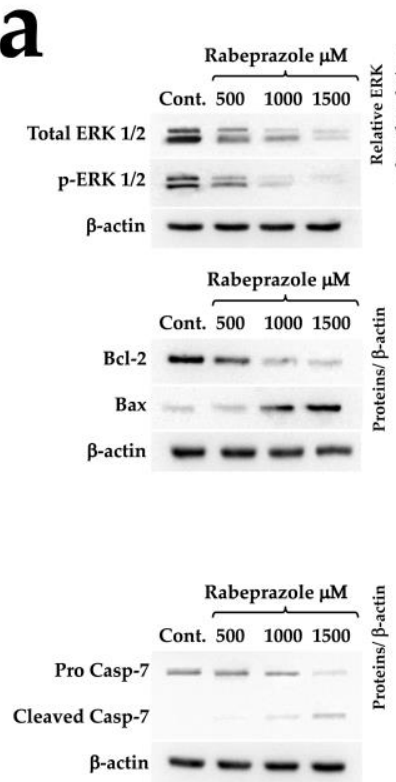
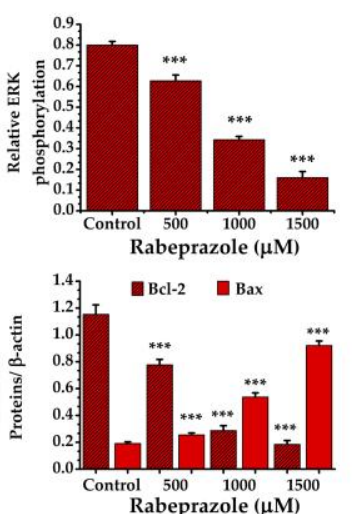

Rabeprazole $(\mu \mathrm{M})$

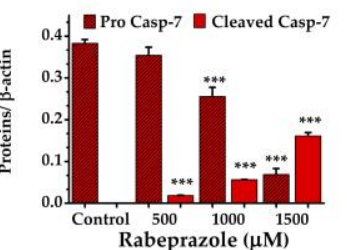

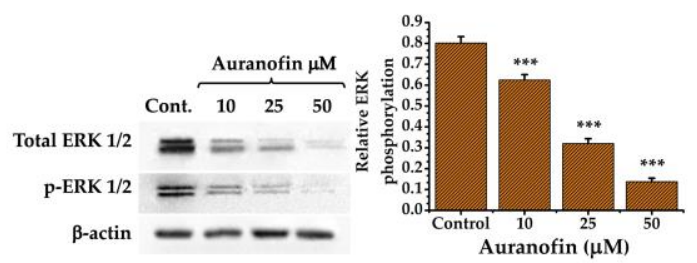
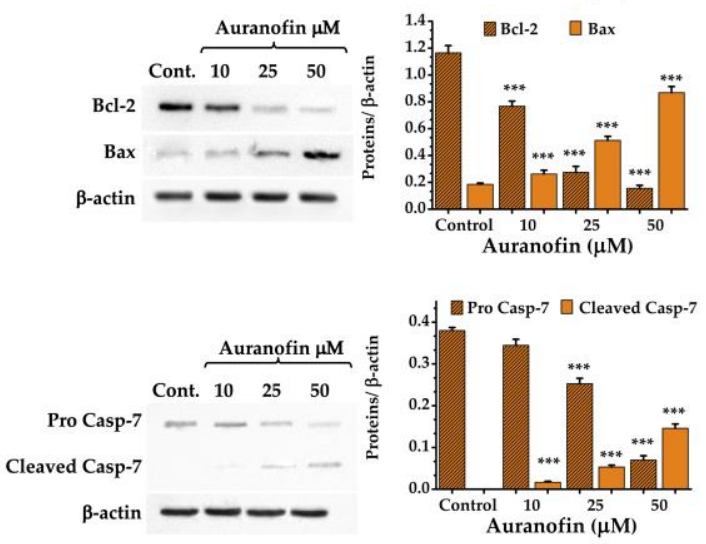

\section{Control}
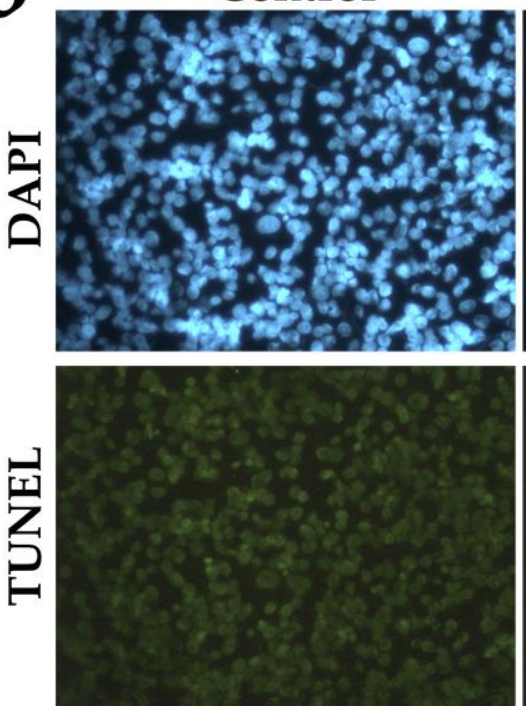

+Rabeprazole
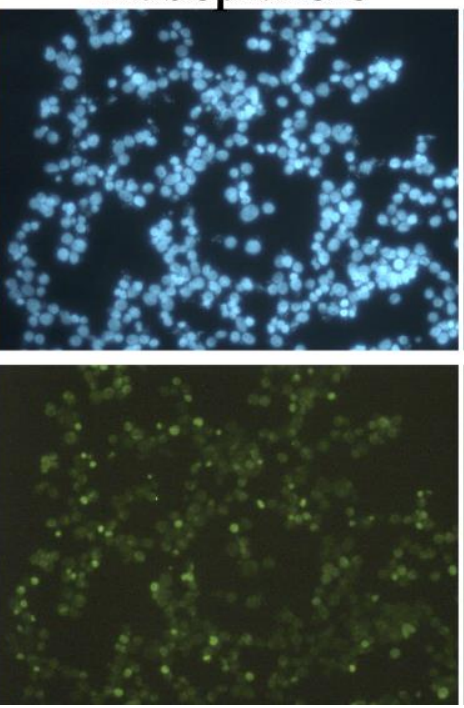

+Auranofin
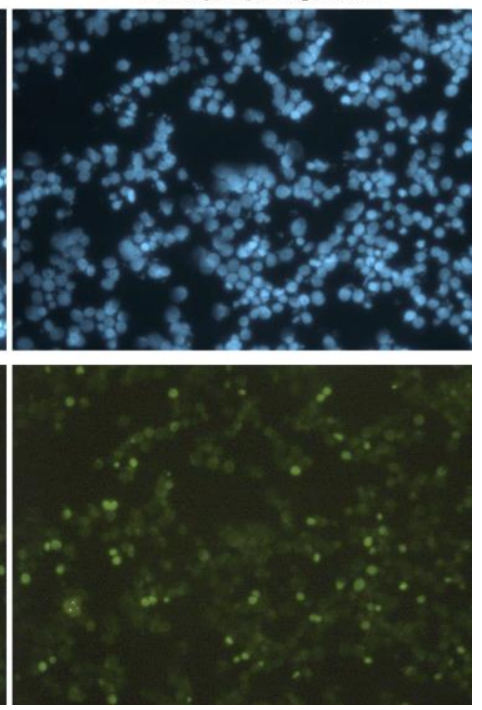

320 Fig. 4. The selective cell death of cancer cells upon drug treatment occurs by means of apoptosis. a,

321 Cancer cells were exposed to $0,500,1000$ and $1500 \mu \mathrm{M}$ rabeprazole and $0,10,25$ and $50 \mu \mathrm{M}$ auranofin.

322 Expression levels of the ERK 1/2 pERK 1/2, Bcl2, Bax, procaspase-7 and caspase-7 proteins are shown. $\beta$ -

323 Actin was used as control. Graphs on the right side of the panels correspond to the results of densitometric

324 analysis of each condition. b, TUNEL assays with cancer cells that were or were not treated with the drugs.

325 Upper panels correspond to DAPI nuclei staining, and the bottom panels correspond to TUNEL assays.

326 Photographs are at $40 \times$. 
328 Deamidated HsTPI is naturally accumulated in tumoral breast cancer tissues and is 329 efficient as a druggable target

330 To determine whether the properties of deamidated HsTPI observed in vitro would translate

331 into an efficient anticarcinogenic strategy, human breast cancer cells were implanted in

332 nude immunocompromised mice and treated with rabeprazole. Tumorigenesis was 333 significantly inhibited by three times weekly treatment with rabeprazole (Fig. 5a) and

334 totally inhibited when pretreated cancer cells were implanted (Fig. 5a, pretreatment). The

335 most notable results from these series of experiments were a) the finding that deamidated

336 HsTPI naturally accumulated in the tumorigenic cells and b) the identical electrophoretic

337 mobility behaviors of tumorigenic HsTPI obtained in vitro and treated with rabeprazole 338 combined with the high production of AGEs in tumors from treated mice (Fig. 5b). 


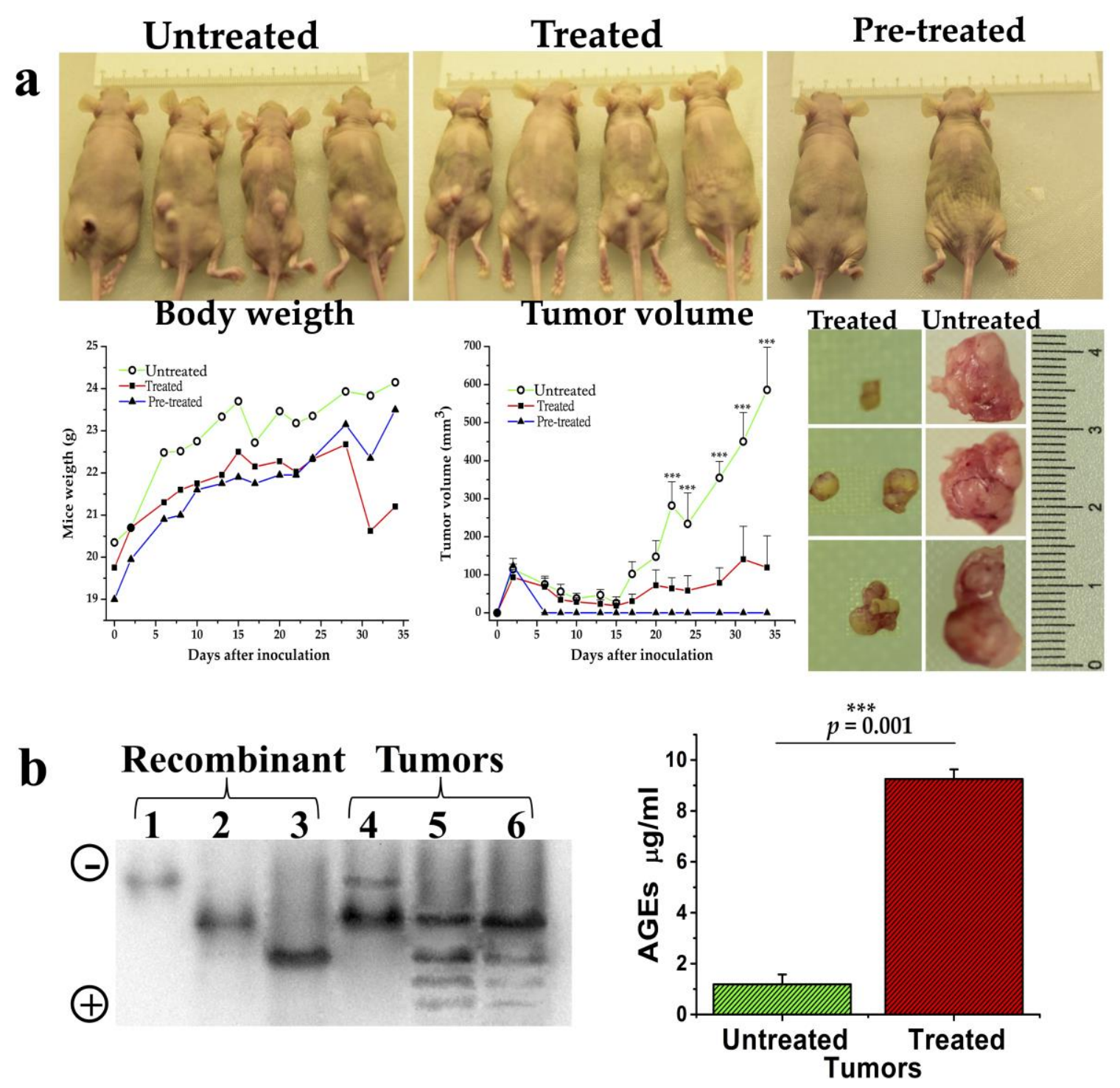

Fig. 5. Deamidated HsTPI accumulated in xenograft tumors, and the antitumorigenic activity of

343 rabeprazole was accompanied by change in HsTPI behavior and the overproduction of AGEs. a,

344 Representative images of mice from the different experimental groups (upper panel). Changes in body weight and tumor volume were noted throughout the experiment (graphs, left bottom). Representative images of tumors (photographs, right bottom) from treated (left) and untreated (right) mice at the end of the experiment clearly depict the antitumorigenic effect of rabeprazole. Mice implanted with breast cancer cells previously incubated for $24 \mathrm{~h}$ with $1 \mathrm{mM}$ rabeprazole (pretreated group) did not develop tumors (upper panel, right). Differences among groups were assessed with one-way ANOVA and Tukey's test with $p$ value $=0.001 * * *$. b, Acidic isoforms of HsTPI obtained from tumors and compared with the recombinant HsTPIs (left panel). Lanes 1-3 show the recombinant proteins and were used as patterns to identify the acidic isoforms corresponding to the nondeamidated (lane 1), once deamidated (lane 2), and twice deamidated HsTPI. Lanes 4-6 show the acidic isoforms of HsTPI from the xenograft tumors of untreated mice (lane 4) and the acidic isoforms of HsTPI from the xenograft tumors of treated mice (lanes 5 and 6). These results clearly show the 
accumulation of deamidated HsTPI in tumors and the effect of treatment with rabeprazole on this protein. The graph (right) shows the significantly increased production of AGEs in tumors treated with rabeprazole compared to untreated tumors. Differences among groups were assessed with one-way ANOVA and Tukey's test with $p$-value $=0.001 * * *$.

\section{Caspase-1 inhibition is related to the accumulation of cellular deamidated HsTPI}

361 To gain more insight in the reason for higher susceptibility of cancer cells for drugs 362 targeting the highly expressed deamidated HsTPI we studied the involvement of caspase-1.

363 HsTPI and some other glycolytic enzymes are substrates of the protease caspase-1[30].

364 According to relative caspase-1 cleavage specificity, the deamidated sequence of HsTPI 365 (X-Asp-Gly-X) is 152 times more prone to be cleaved by Caspase- 1 than its nondeamidated counterpart (X-Asn-Gly-X)[31, 32]. In addition, caspase- 1 mRNA expression was found to

367 be significantly decreased in the breast cancer tissues of patients, and treatment with a 368 caspase-1 inhibitor markedly increased the proliferative and invasive abilities of 369 MDA-MB-231 cells[33]. The keystone of our hypothesis implicates the accumulation of 370 deamidated HsTPI in cancer cells. Therefore, we wondered whether caspase-1 plays a role 371 on the absence of deamidated HsTPI in normal cells in contrast with its accumulation in 372 cancer cells. Remarkably, normal cells treated with a caspase-1 inhibitor showed the de 373 novo accumulation of acidic isoforms similar to those previously found in cancer cells (Fig. 374 6a, lanes 4 and 5). Additionally, caspase-1 activity was inhibited by 77\%, whereas HsTPI 375 activity was increased by $45 \%$ in the noncancer cells (Suppl. Table 3). Moreover, the acidic 376 isoforms of HsTPI from these cells not only adopted the behavior of their counterparts in 377 cancer cells but also had become sensitized to rabeprazole and auranofin (Fig. 6a, upper 378 and bottom, lanes 6 and 7). These normal cells with inhibited caspase-1 activity underwent 379 the aforementioned changes and significantly increased their capacity to produce advanced 380 glycation end products (AGEs) when they were treated with rabeprazole or auranofin (Fig. 381 6b). Based on these variables, these results show how normal cells can be guided toward 382 "cancer-like" behavior regarding glycolysis, showing characteristic HsTPI electrophoretic 383 profiles, and leading to selective cell apoptosis (Fig. 6 c). Altogether, the results clarify the 384 mechanisms involved in natural accumulation of deamidated HsTPI in cells, leading to the 385 generation of a selective target. 

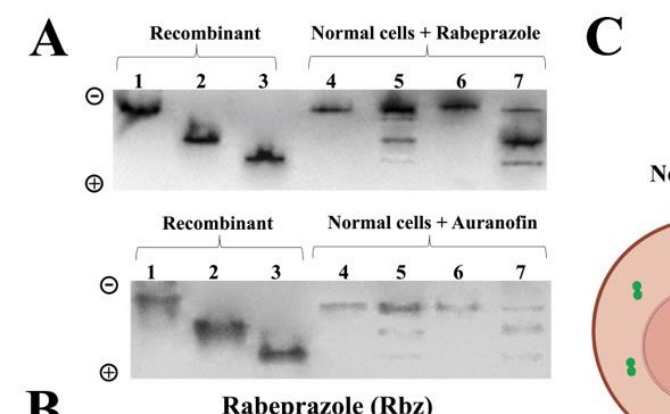

\section{B}
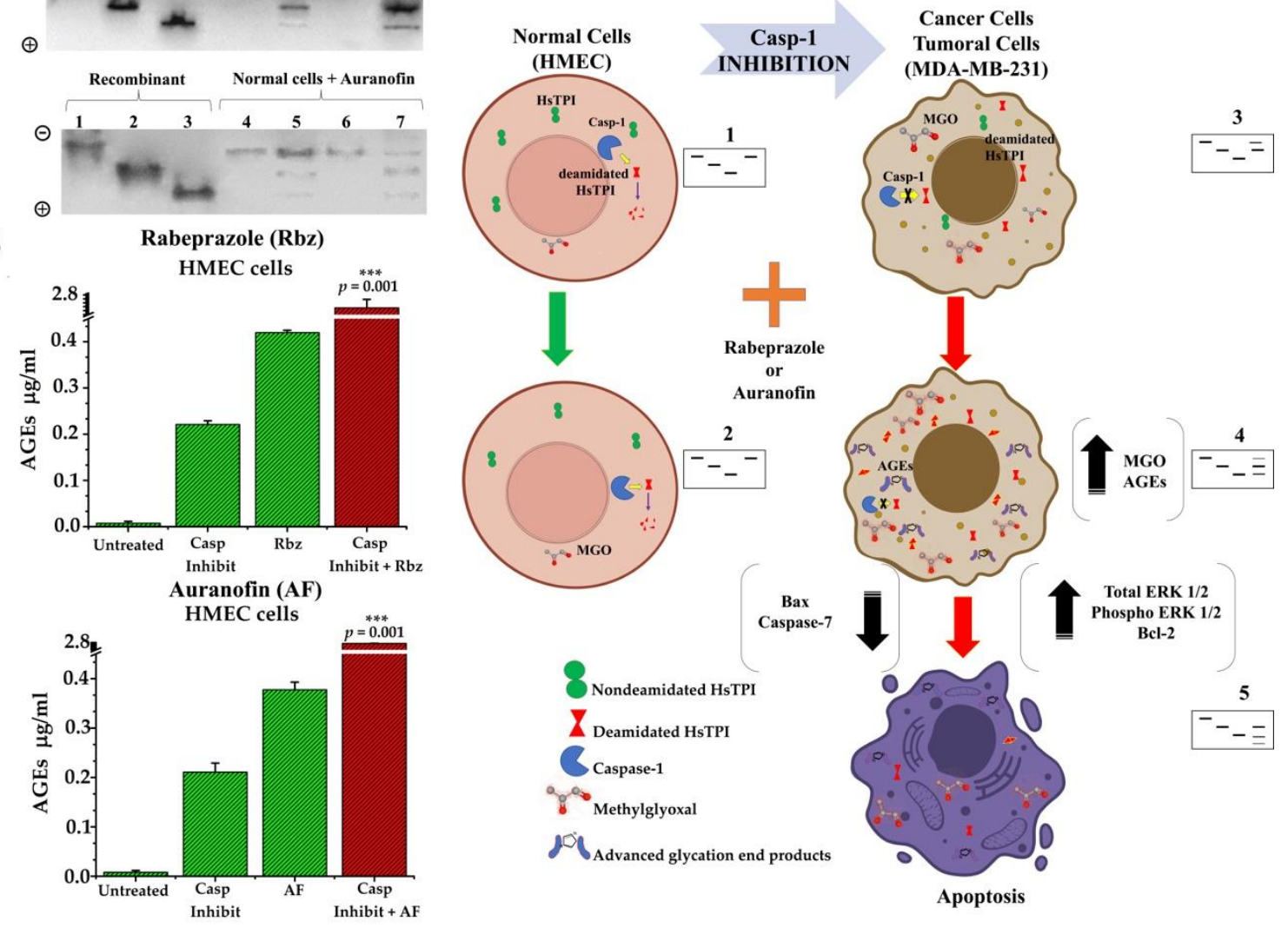

Figure. 6. Caspase-1 inhibition in normal cells mimics cancer cells phenotype. (A) nPAGE and western blot analysis of HMECs with or without treatment with a caspase-1 inhibitor. Lanes 1 to 3 (10 $\mu \mathrm{g} / \mathrm{lane})$ : recombinant nondeamidated, once deamidated, and double deamidated HsTPI, respectively. Lanes 4 to 7 (100 $\mu \mathrm{g}$ protein/lane): proteins from the cellular extract of normal cells (HMECs). Lane 4: cells that were untreated with the caspase-1 inhibitor (top, rabeprazole and bottom, auranofin); lane 5 (top and bottom): cells treated with the caspase-1 inhibitor; lane 6: cells that were treated with rabeprazole (top) and auranofin (bottom), lane 7: cells that were pretreated with the caspase-1 inhibitor and treated with rabeprazole (top) and auranofin (bottom). (B) AGEs production in normal cells (HMEC) without the caspase-1 inhibitor, cells that were treated with the caspase-1 inhibitor (Casp Inhibit), and cells that were pretreated with the caspase-1 inhibitor and treated with rabeprazole (upper) and auranofin (bottom). Significant differences were obtained for cells pretreated with the caspase-1 inhibitor and treated with either rabeprazole or auranofin, $p=0.001 * * *$. $(\mathbf{C})$ Schematic illustration of the role of deamidated HsTPI and caspase-1 in normal and cancerous phenotypes (i.e., MDA-MB-231 cells and tumors). 1, 2, 3, 4, and 5 represent the corresponding nPAGE profiles of HsTPI acidic isoforms from each cell phenotype. 


\section{DISCUSSION.}

405 Here we report the selective effect of rabeprazole and auranofin on HsTPI in vitro, in situ, 406 and in vivo. The derivatization of Cys residues guides the effects exerted by these drugs. 407 Cancer targets are usually selected based on their enhanced expression or even their 408 overactivity [34, 35]. On this basis, human TIM fulfills one requirement for use as a drug 409 target as it is upregulated in cancer cells $[4,36]$. Nevertheless, this protein is present in all 410 cells regardless of whether they are cancerous, which significantly diminishes the chosen 411 drug's cellular selectivity. To address this issue, we propose a new approach to enhancing 412 drug selectivity based on the distinctive characteristics of deamidated HsTPI. First, the 413 presence of deamidated HsTPI in cancer cells but not their normal counterparts. Second, 414 deamidated HsTPI accumulates in cancer cells. Third, deamidated HsTPI is involved in 415 cell-specific cell death induced by rabeprazole and auranofin.

416 HsTPI is a potential target in cancer mainly by using inhibitors directed to its active site or 417 factors that diminish its expression [37]. Nonetheless, these proposals' main problem is that 418 the molecular elements involved in the likely therapeutic strategies are present at both 419 pathologic and normal stages. Consequently, an essential disadvantage of the latter 420 approach is that it vastly reduces the safety of such methods. Herein, we have demonstrated 421 that deamidated HsTPI is a distinctive and conspicuous target in triple-negative breast 422 cancer cells but not in their noncancerous counterparts. Hence, our proposal would help the 423 drug design pipeline in the pharmaceutical industry and increase clinical trials' success rate. 424 MDA-MB-231 cells are triple-hormone receptor-negative breast cancer cells characterized 425 by the minimal expression of estrogen and progesterone receptors and absence of human 426 epidermal growth factor receptor-2 (HER2). Because these cancer cells lack HER2, 427 hormone therapy and drugs targeting HER2 are not helpful, leaving chemotherapy as the 428 main systemic treatment option for triple-negative breast cancer. The primary therapies 429 used for this cancer combine the drugs adriamycin and cyclophosphamide; adriamycin, 430 cyclophosphamide, and paclitaxel; and docetaxel and cyclophosphamide [38]. Since these 431 drugs are directed to targets (mainly nucleic acids and the replication machinery) present in 432 both cancer and normal cells, the side effects of such treatments are considerable and 433 usually severe. 
434 Additionally, their economic burden has a significant impact on patients suffering from this

435 disease [39]. In contrast, our strategy would represent a safer and less expensive therapy for

436 this kind of cancer.

438 It is important to note that the two different drugs used yielded the same output, especially 439 that caused by the effects on deamidated HsTPI. Accordingly, both treatments exert 440 selective cellular death, mainly attributable to the presence of deamidated HsTPI and its 441 impact on these cancer cells. Consequently, glycolytic flux diminishes, and the 442 concentrations of MGO and AGEs concomitantly increase. Although energy metabolism 443 was affected, the leading cause of selective cell death was the toxic effect of MGO 444 overproduction, as shown in other diseases [40, 41]. Emerging evidence indicates that $445 \mathrm{MGO}$ and AGEs can induce the apoptosis process [42, 43].

447 Since the response of cancer cells in tumors is affected by factors such as their 448 heterogeneity, components of the microenvironment, and anatomical structures for proper 449 growth [44], the xenograft model was of utmost importance. In this preclinical scenario, the 450 protein concerned was corroborated by the accumulation of acidic isoforms equivalent to 451 deamidated HsTPI in all tumors. Collectively, these results in tumors faithfully mimicked 452 the behavior of HsTPI demonstrated in cell lines. Other proton pump inhibitors (PPIs) have 453 been reported to act as tumor suppressors [6, 45], with proteins upregulated in cancer stages 454 acting as possible targets; however, their most significant weakness is that the proposed 455 targets are found in cancer and normal cells.

456 The role of deamidated HsTPI as a triple-negative breast cancer-specific target was 457 supported by the change in phenotype of normal cells (HMECs) to a cancer phenotype, 458 based on the described HsTPI behavior. This was achieved by inhibiting cellular caspase-1 459 activity in HMECs. Most importantly, these cells were sensitized to the drug treatments. 460 Indeed, we previously demonstrated in a cell model that the presence of deamidated HsTPI 461 alone caused sensitization to another PPI, impairing cell growth[10].

462 The strategy stated here highlights the posttranslational modifications favored in cancer 463 cells, which naturally generate cancer-specific targets. Based on the knowledge acquired 464 herein, pathologic cells should contain a collection of proteins with special skills similar to 
465 those shown by HsTPI. That is, structural signatures that usually promote changes in 466 proteins that drive their dead-end elimination (i.e., protein turnover) in normal cells but 467 translate into new functions that lead to adaptive advantages in pathological cells. MGO is 468 a hormetic metabolite related to tumor growth and metastasis [46, 47]. In fact, in this effect, 469 we found a hidden opportunity to push the triple-negative cancer cells to overproduce 470 MGO and commit them to suicide (apoptosis), achieving a novelty and safer strategy on the 471 therapeutics for such highly aggressive cancer. In contrast, cell death would not be as 472 specific for malignant cells if toxic metabolites were administered exogenously. Other 473 types of cancer or even to other pathologies could also adapt our approach for different 474 proteins that meet the characteristics described herein.

476 According to our findings, three main hallmarks might guide the search for other types of 477 cancers susceptible to being treated using the strategy studied herein - the increase in 478 glycolytic flux, the upregulated HsTPI, and downregulated Casp-1. The presence of all 479 these factors, the coexistence of two of them, or the presence of one of them could indicate 480 the natural accumulation of deamidated HsTPI. In this regard, many cancers might be 481 candidates since they show upregulation of HsTPI (for a detailed list, see [48]). Indeed, the 482 HsTPI upregulation can provide non-deamidated and deamidated HsTPI to the cells as we 483 observed in MDA-MB-231, which is likely reported by others as two types of HsTPIs when 484 they analyzed the proteome of lung squamous carcinoma [49].

486 Interestingly, monocytic lineage of acute leukemia was previously reported with highly 487 expressed HsTPI [50], and our preliminary findings working with acute leukemia cells 488 show similar results as those in triple-negative breast cancer. We are still working on this to 489 better understand and translate our strategy to a clinical trial shortly. 


\section{Methods}

Reagent and general materials.

497 Luria-Bertani (LB) medium and isopropyl- $\beta$-D-thiogalactopyranoside (IPTG) were 498 purchased from VWR Life Science Products (Radnor, Pennsylvania, USA). Glycerol-3499 phosphate dehydrogenase $(\alpha-G D H)$, L-lactate dehydrogenase from rabbit muscle, and 500 reduced nicotinamide adenine dinucleotide (NADH) were obtained from Roche (Penzberg, 501 Upper Bavaria, Germany). Immobilized metal affinity chromatography (IMAC) resin was 502 obtained from Bio-Rad (Hercules, California, USA). Sephadex G-25 Fine Resin was 503 obtained from Amersham Biosciences (Amersham, UK). Amicon Ultra $30 \mathrm{kDa}$ filters were 504 purchased from Merck-Millipore Corporation (Billerica, Massachusetts, USA). Fetal 505 bovine serum (FBS), penicillin, streptomycin and trypsin EDTA solutions were purchased 506 from Invitrogen (Carlsbad, USA). The other reagents mentioned were acquired from 507 Sigma-Aldrich (St. Louis, MO, USA).

\section{Molecular docking studies of deamidated and nondeamidated HsTPI.}

509 To carry out the docking studies, the crystallographic structures of HsTPI WT 510 (nondeamidated) and HsTPI N16D (deamidated) that had been deposited in the Protein 511 Data Bank (PDB) were downloaded. Atomic coordinates of the nondeamidated and 512 deamidated proteins (PDB IDs: 2JK2 and 4UNK, respectively) were prepared by removing 513 all water molecules and heteroatoms with PyMOL version 2.0.7 (Schrödinger Inc, NY, 514 USA). The structures were energy minimized with Chimera software [51], and the new 515 coordinates were used for docking calculations. Structures of the drugs rabeprazole and 516 auranofin were obtained from the PubChem Compound Database 517 (https://pubchem.ncbi.nlm.nih.gov) and energy minimized with Avogadro version 1.2. 518 Structures of the nondeamidated and deamidated HsTPIs were prepared by adding 519 hydrogen atoms and Kollman charges (6.00 and 3.999, respectively) with AutoDock Tools 520 (ADT) version 1.5.6 [52]. The molecular docking program AutoDock Vina version 1.1.2 521 [53] was used with the default settings, and the output files were saved in pdbqt format. 522 Protein receptors and ligands were converted into pdbqt format. The ligand-binding site 523 was defined as the interface of the dimer. After docking, close interactions for binding of 524 the target with the ligands were analyzed and visualized using ADT and PyMOL version 
Expression and purification of recombinant enzymes.

528 The genes encoding wild-type and mutant (WT, N16D and N16D/N72D) HsTPI were 529 cloned into the vector pET3a-HisTEV as previously reported [12]. The plasmid provides 530 six histidine residues (His6) at the N-terminus of the protein and a tobacco etch virus 531 protease (TEVp) recognition sequence that facilitates protein purification. The plasmids 532 containing inserts (pET3a-HisTEV-wt-HsTPI, pET3a-HisTEV-N16D-HsTPI and pET3a533 HisTEV-N16D/N72D-HsTPI) were used to transform the Escherichia coli BL21534 CodonPlus-RIL strain. The overexpression and purification of recombinants WT 535 (nondeamidated HsTPI), N16D HsTPI (deamidated HsTPI) and N16D/N72D HsTPI 536 (double deamidated HsTPI) were carried out as previously described [12]. Purified proteins 537 were ultrafiltered with Centricon filters (cutoff of $30 \mathrm{kDa}$ for WT and $10 \mathrm{kDa}$ for N16D and $538 \mathrm{~N} 16 \mathrm{D} / \mathrm{N} 72 \mathrm{D})$ until the volume reached $0.5 \mathrm{~mL}$, which was repeated 3 times after the 539 addition of $5 \mathrm{~mL}$ of buffer containing $100 \mathrm{mM}$ triethanolamine and $10 \mathrm{mM}$ EDTA (pH 7.4) 540 (TE buffer). Finally, the concentrated proteins were precipitated with ammonium sulfate at $54175 \%$ saturation and maintained at $4{ }^{\circ} \mathrm{C}$. To remove the His ${ }_{6}$-TEV tag, the protein 542 suspension was centrifuged at $12,000 \mathrm{rpm}$ for $20 \mathrm{~min}$ at $4{ }^{\circ} \mathrm{C}$, and the pellet was 543 resuspended in $50 \mathrm{mM}$ Tris ( $\mathrm{pH} 8.0), 0.5 \mathrm{mM}$ EDTA and incubated at room temperature for $54416 \mathrm{~h}$ in the presence of the protease TEVp at 1:50 (w/w) (protease/HsTPI) and $1 \mathrm{mM}$ 545 dithiothreitol (DTT). Thereafter, the incubated sample was loaded into a column containing 546 IMAC resin previously equilibrated with $100 \mathrm{mM}$ triethanolamine ( $\mathrm{pH}$ 7.4). Enzymes 547 without the His 6 -TEV tag were recovered, ultrafiltered, precipitated with ammonium sulfate 548 and stored at $4{ }^{\circ} \mathrm{C}$ until use. To remove the precipitating agent, the protein was centrifuged 549 as mentioned and suspended in TE buffer. The protein concentration was calculated 550 spectrophotometrically at $280 \mathrm{~nm}$ with an extinction coefficient of $\varepsilon=33,460 \mathrm{M}^{-1} \mathrm{~cm}^{-1}$ 551 [54]. The purity and integrity of the proteins were verified by sodium dodecyl sulfate 552 polyacrylamide gel electrophoresis (16\% SDS-PAGE), and proteins were stained with 553 colloidal Coomassie brilliant blue.

554 Prior to any assays, the recombinant enzymes were equilibrated in TE buffer and incubated 555 in the presence of $5 \mathrm{mM}$ DTT for $30 \mathrm{~min}$ at $4{ }^{\circ} \mathrm{C}$. To remove the reducing agent, the protein 
556 was spin filtered in a 1-mL column loaded with Sephadex G-25 Fine Resin previously

557 equilibrated with TE buffer, and the protein concentration was estimated by the absorbance

558 at $280 \mathrm{~nm}$.

Inactivation assays with nondeamidated (WT) and deamidated (N16D) HsTPI treated with rabeprazole and auranofin.

562 Recently, we demonstrated that the PPI omeprazole selectively inactivates deamidated 563 HsTPI [10]. Furthermore, in a previous work, we showed that among commonly used PPIs 564 (benzimidazole derivatives), rabeprazole was the most efficient PPI in inactivating the TIM 565 of Giardia lamblia [55]. Therefore, we chose rabeprazole based on the latter finding; 566 additionally, we tested auranofin to compare the effects of rabeprazole with those of a non567 PPI drug. A $100 \mathrm{mM}$ stock solution of rabeprazole was prepared; to acid activate it, the 568 solution was solubilized in $20 \%$ dimethyl sulfoxide and $5 \% 0.1 \mathrm{~N} \mathrm{HCl}$, incubated for 30 $569 \mathrm{~min}$ at room temperature in the dark, and diluted with TE buffer to obtain a $5 \mathrm{mM}$ solution.

570 A $100 \mathrm{mM}$ stock of auranofin was prepared by dissolving auranofin in 100\% ethanol, and 571 serial dilutions were made with TE buffer. For the inactivation assays, the recombinant 572 enzymes were incubated at $0.5 \mathrm{mg} \cdot \mathrm{mL}^{-1}$ for $2 \mathrm{~h}$ at $37^{\circ} \mathrm{C}$ in the presence of $0,10,25,50$, 573100 and $250 \mu \mathrm{M}$ rabeprazole or auranofin. After incubation, the samples were diluted, and $5745 \mathrm{ng} \cdot \mathrm{mL}^{-1}$ and $50 \mathrm{ng} \cdot \mathrm{mL}^{-1}$ samples of WT (nondeamidated) and N16D (once deamidated), 575 respectively, were taken to measure their enzymatic activity. Enzyme activity was 576 spectrophotometrically measured (Spectrophotometer Cary 50, Agilent Technologies, CA, 577 USA) by following DHAP synthesis with a coupled system that followed the oxidation of $578 \mathrm{NADH}$ at $340 \mathrm{~nm}$ [56]. The results represent the mean of four independent experiments and 579 are expressed as the percent activity versus drug concentration, with the activity of the enzyme without the drug set at $100 \%$.

\section{Quantification of free thiols in recombinant enzymes.}

583 Because the principal mechanism of action of the drugs employed here (rabeprazole and 584 auranofin) is thought to be the derivatization of Cys residues, the number of derivatized 585 Cys residues in the recombinant enzymes was determined by using Ellman's reagent (5,5'586 dithiobis-(2-nitrobenzoic) acid, DTNB) [57]. To carry out the experiment described above, 
$587 \quad 0.5 \mathrm{mg} \cdot \mathrm{mL}^{-1}$ protein was incubated without or with 250 and $50 \mu \mathrm{M}$ rabeprazole and 588 auranofin, respectively, for $2 \mathrm{~h}$ at $37^{\circ} \mathrm{C}$. After the incubation period, the proteins were 589 extensively washed by ultrafiltration with Centricon filters to eliminate excess drug, and the 590 protein concentration was estimated by determining the absorbance at $280 \mathrm{~nm}$. Next, an 591 aliquot was taken and used to evaluate the residual activity of assayed proteins. The free 592 thiol (Cys) content of the samples was spectrophotometrically quantified as follows: the 593 basal absorbance of $1 \mathrm{mM}$ DTNB and 5\% SDS dissolved in TE was measured at $412 \mathrm{~nm}(\varepsilon$ $\left.594412 \mathrm{~nm}=14.1 \mathrm{mM}^{-1} \cdot \mathrm{cm}^{-1}\right)$, and the increase in absorbance following the addition of $200 \mu \mathrm{g}$ of 595 protein was monitored. The number of derivatized Cys residues was indirectly calculated 596 by subtracting the number of free Cys residues in the derivatized enzyme (treated with 597 rabeprazole or auranofin) from the number of free Cys residues in the enzyme in the 598 absence of the drugs. The results represent the mean of at least four independent 599 experiments.

601 Determination of fluorescence emission spectra of the recombinant enzymes.

602 The intrinsic and extrinsic fluorescence emission spectra of the proteins were measured 603 using an LS55 spectrofluorometer (Perkin Elmer, Waltham MA, USA). WT or N16D 604 HsTPI $\left(0.5 \mathrm{mg} \cdot \mathrm{mL}^{-1}\right)$ was incubated for $2 \mathrm{~h}$ at $37{ }^{\circ} \mathrm{C}$ with or without 250 and $50 \mu \mathrm{M}$ 605 rabeprazole and auranofin, respectively. Next, the proteins were extensively washed by 606 ultrafiltration with Centricon filters to eliminate excess drug, and their concentration were 607 recalculated by measuring their absorbance at $280 \mathrm{~nm}$. To determine the intrinsic 608 fluorescence, the enzymes $\left(0.5 \mathrm{mg} \cdot \mathrm{mL}^{-1}\right)$ were excited at $295 \mathrm{~nm}$, and the fluorescence 609 emission spectra were recorded from 300 to $500 \mathrm{~nm}$. To determine the extrinsic 610 fluorescence, a stock solution of $15 \mathrm{mM}$ ANS dissolved in methanol was prepared. Then, $611100 \mu \mathrm{M}$ ANS was added to the samples (maintained with slow agitation), which were 612 excited at $385 \mathrm{~nm}$, and the fluorescence emission spectra were monitored from 400 to 600 $613 \mathrm{~nm}$. For each sample reading, the background fluorescence was subtracted (buffer with 614 drug, buffer with ANS, or buffer with ANS and rabeprazole or auranofin). Each spectrum is 615 the average of three scans. The results represent the mean of four independent experiments 616 and are expressed as the percent fluorescence intensity versus wavelength. 


\section{Cell culture and identification of cellular deamidated HsTPI by the selective cleavage}

619 of Asn-Gly.

620 For general cell culture procedures, the MDA-MB-231 (breast cancer cells) cell line was 621 obtained from American Type Culture Collection (ATCC; Rockville, MD, USA) and 622 maintained in DMEM supplemented with 10\% FBS and antibiotics (100 U/mL penicillin 623 and $100 \mathrm{mg} / \mathrm{mL}$ streptomycin). Acting as noncancerous (control) cells, HMECs were 624 obtained from ATCC and cultured in mammary epithelial cell basal medium (ATCC \#PCS625 600-030) supplemented with components of the mammary epithelial growth kit (ATCC 626 \#PCS-600-040). For all experiments, both cell lines were used from passages 2-5 and 627 cultured at $37{ }^{\circ} \mathrm{C}$ in a humidified $5 \% \mathrm{CO}_{2}$ atmosphere, detached with $0.05 \%$ trypsin in 0.53 $628 \mathrm{mM}$ EDTA, incubated for $5 \mathrm{~min}$ at $37{ }^{\circ} \mathrm{C}$, centrifuged at $2000 \mathrm{rpm}$ for $5 \mathrm{~min}$ and washed 629 three times with phosphate-buffered saline (PBS).

630 Since the amino acid sequence of HsTPI contains two Asn-Gly pairs, both cell lines were 631 properly maintained and used to determine the deamidation of cellular HsTPI by selective 632 cleavage with hydroxylamine [18]. A validated method for peptide mapping and sequence 633 analysis of Asn-Gly pairs[19], this method is based on placing the proteins under alkaline 634 conditions (i.e., $\mathrm{pH}$ 9.0) to deprotonate the amide group of the Asn residue, which in turn 635 exert a nucleophilic attack on the adjacent Gly residue located in the C-terminus. This 636 results in the formation of succinimide, which is selectively cleaved in the presence of an 637 excess of hydroxylamine. Notably, deamidated proteins contain Asp (or isoAsp) instead of 638 Asn; thus, under the conditions described for this method, succinimide is not formed, 639 preventing cleavage by hydroxylamine [20].

640 HMECs $\left(1 \times 10^{7}\right)$ and MDA-MB-231 cells were incubated for $24 \mathrm{~h}$ under the conditions 641 mentioned above. After washing, the cells were lysed in ice-cold RIPA lysis buffer 642 containing protease inhibitors (sc-24948, Santa Cruz Biotechnology, Santa Cruz, CA, 643 USA) and centrifuged at 12,000 rpm for $20 \mathrm{~min}$ at $4{ }^{\circ} \mathrm{C}$. The supernatant was collected, and 644 the protein content was quantified by Bradford assay. The protein extracts from each 645 culture were immunoprecipitated with anti-human TIM (H-11) (Santa Cruz Biotechnology) 646 for $1 \mathrm{~h}$ at $4{ }^{\circ} \mathrm{C}$. After this, protein A/G agarose (Santa Cruz Biotechnology, sc-2003) was 647 added to the mixtures, which were adequately resuspended and incubated overnight at $4{ }^{\circ} \mathrm{C}$. 648 The mixtures were centrifuged at $2000 \mathrm{rpm}$ for $3 \mathrm{~min}$ and washed with PBS 7 times. The 
649 immunoprecipitation products were washed with $0.2 \mathrm{M}$ glycine- $\mathrm{HCl}$ to separate the protein

$650 \mathrm{~A} / \mathrm{G}$ plus protein-antibody-protein complex. To obtain the isolated cellular HsTPI, samples

651 were centrifuged at $2000 \mathrm{rpm}$ for $5 \mathrm{~min}$, immediately after which the supernatant was

652 taken, and the $\mathrm{pH}$ was neutralized with Tris buffer $(\mathrm{pH} \mathrm{8.0)}$.

653 Hydroxylamine cleavage of recombinant and cellular HsTPI was performed as follows. WT 654 (nondeamidated), N16D (once deamidated) and N16D/N72D (twice deamidated) 655 recombinant HsTPI enzymes and cellular HsTPI $(1 \mathrm{mg} / \mathrm{mL})$ were separately incubated for 8 $656 \mathrm{~h}$ at $45{ }^{\circ} \mathrm{C}$ with $2 \mathrm{M}$ hydroxylamine- $\mathrm{HCl}$ and $1 \mathrm{M}$ sodium carbonate $\left(\mathrm{Na}_{2} \mathrm{CO}_{3}\right), \mathrm{pH} 9.0$. 657 Immediately after incubation, the samples were loaded on a 16\% SDS-PAGE gel. To 658 analyze the patterns of HsTPI hydrolysis, gels were stained with colloidal Coomassie 659 brilliant blue. The assays were carried out in triplicate to guarantee reproducibility.

660 Cell line authentication.

661 Cell line authentication was performed by ATCC, sales order SO0359144, FTA Barcode 662 STRA11162. Briefly, seventeen short tandem repeat (STR) loci plus gender determining 663 locus, Amelogenin, were amplified using commercially available PowerPlex ${ }^{\circledR}$ 18D Kit fro 664 Promega. The cell line samples were processed using ABI Prism ${ }^{\circledR} 3500 x \mathrm{x}$ Genetic 665 Analyzer. Data were analyzed using GeneMapper ${ }^{\circledR}$ ID-X v1.2 software (Applied 666 Biosystems). Appropriate positive and negative controls were run and confirmed for each 667 sample.

668 Native gel electrophoresis and western blot analysis of recombinant enzymes, protein 669 cellular extracts, and tumors.

670 The HsTPI isoforms from cellular extracts were identified with nPAGE combined with 671 western blot analysis using the monoclonal antibody anti-TIM (H11). HMECs or MDA672 MB-231 cells $\left(1 \times 10^{7}\right)$ were exposed to $0,500,1000$ and $1500 \mu \mathrm{M}$ rabeprazole and $0,25,50$ 673 and $100 \mu \mathrm{M}$ auranofin for $24 \mathrm{~h}$ under the abovementioned conditions. Cells were lysed in 674 ice-cold RIPA lysis buffer containing protease inhibitors. Protein quantification was 675 performed by the Bradford method. nPAGE gels were loaded with $1 \mu \mathrm{g} /$ lane recombinant 676 nondeamidated, N16D (once deamidated), and N16D/N72D (twice deamidated) HsTPI 677 proteins and $100 \mu \mathrm{g} / \mathrm{lane}$ cellular protein extracts. nPAGE gels were prepared with $7 \%$ 678 polyacrylamide and Tris-glycine buffer at $\mathrm{pH} 8.5$ [58]. Samples were mixed with native 
679 buffer and run at $7 \mathrm{~mA}$ and $4{ }^{\circ} \mathrm{C}$ for $3 \mathrm{~h}$. Proteins were transferred to polyvinylidene 680 difluoride (PVDF) membranes $\left(0.8 \mathrm{~mA} / \mathrm{cm}^{2}, 2 \mathrm{~h}\right)$ in $25 \mathrm{mM}$ Tris buffer containing $192 \mathrm{mM}$ 681 glycine and 20\% methanol. The membrane was blocked for $1 \mathrm{~h}$ with Tris-buffered saline 682 with $0.1 \%$ Tween-20 (TBS-T) supplemented with 5\% bovine serum albumin (BSA), 683 washed one time with TBS-T and incubated overnight at $4{ }^{\circ} \mathrm{C}$ with anti-TIM (H-11) diluted 684 1:3000 in TBS-T containing 1\% BSA and washed three times with the same buffer. An 685 anti-mouse IgG secondary antibody (diluted 1:5000) conjugated with horseradish 686 peroxidase (HRP) was used to reveal the immunoblot bands by chemiluminescence (Clarity 687 Western ECL substrate, Bio-Rad) following the supplier's instructions. Blot image 688 acquisition was performed using a ChemiDoc XRS+ system (Bio-Rad Laboratories, Inc., 689 USA).

690 nPAGE and immunoblotting in tumors were performed as follows. Tumors from nude mice 691 were obtained by dissection and immediately frozen with liquid nitrogen. Afterwards, they 692 were macerated with a mortar until pulverized, immediately after which they were 693 resuspended in PBS and treated as described above. All the assays were carried out in 694 triplicate.

Cell proliferation and enzymatic assays to assess cultured cells under drug treatment.

697 HMECs and MDA-MB-231 cells were exposed to drugs, after which cell proliferation and 698 the inactivation of cellular HsTPI were determined as follows. A total of $1 \times 10^{5}$ cells/well in 699 a final volume of $250 \mu \mathrm{L}$ in six-well plates were exposed to $0,100,250,500,1000$ and $7001500 \mu \mathrm{M}$ rabeprazole and $0,10,25,50,100,250$ and $500 \mu \mathrm{M}$ auranofin for $24 \mathrm{~h}$ under the 701 culture conditions mentioned above. The cells were detached, washed, and quantified with 702 a hemocytometer. Cell proliferation was measured with 3-(4,5-dimethylthiazol-2-yl)-2,5703 diphenyltetrazolium bromide (MTT). HMECs or MDA-MB-231 cells $\left(1 \times 10^{3}\right)$ were mixed 704 in $100 \mu \mathrm{L}$ of PBS per well in a 96-well plate, and $10 \mu \mathrm{L}$ of MTT was added and incubated 705 for $4 \mathrm{~h}$ in the dark. The formed formazan crystals were dissolved in DMSO, and the 706 absorbance at $570 \mathrm{~nm}$ was measured in an Epoch microplate spectrophotometer (BioTek, 707 Vermont, USA) [59]. The results are shown as the mean of four independent experiments 708 and presented as the percent viability vs drug treatment; the viability measured with cellular 709 extracts without treatment was set at $100 \%$. To measure cellular HsTPI enzyme activity, the 
710 coupled assay system described above was used. HMECs or MDA-MB-231 cells exposed

711 to drugs were detached, washed and resuspended in TE buffer. Cell suspensions were lysed

712 with 5 freeze/thaw cycles (liquid nitrogen and $40{ }^{\circ} \mathrm{C}$ ). Then, the protein concentration was

713 determined by Bradford assay. For enzymatic assays, 40- $\mu$ g protein samples from the

714 cellular extracts were added to $500 \mu \mathrm{L}$ of enzymatic reaction mixture and

715 spectrophotometrically assessed at $340 \mathrm{~nm}$. The results are presented as the percent activity

$716 v s$ drug treatment, and TIM enzyme activity in cellular extracts without treatment was set at

$717100 \%$.

\section{L-Lactate measurements.}

720 Extracellular L-lactate levels were determined by measuring the reduction of $\mathrm{NAD}^{+}$ 721 to NADH by L-lactate dehydrogenase according to Bergmeyer et al., 1975 [60]. HMECs 722 and MDA-MB-231 cells $\left(5 \times 10^{6}\right)$ were treated with 0,700 and $1000 \mu \mathrm{M}$ rabeprazole and 0 , 72370 and $150 \mu \mathrm{M}$ auranofin for $24 \mathrm{~h}$ under the abovementioned culture conditions. Then, the 724 cells were extensively washed with Krebs-Ringer buffer $(125 \mathrm{mM} \mathrm{NaCl}, 5 \mathrm{mM} \mathrm{KCl}, 25$ $725 \mathrm{mM}$ HEPES $1.4 \mathrm{mM} \mathrm{CaCl}_{2}, 1 \mathrm{mM} \mathrm{KH} \mathrm{PO}_{4}, 1 \mathrm{mM} \mathrm{MgCl}$, $\mathrm{pH}$ 7.4) and resuspended in 250 $726 \mu \mathrm{L} / 1 \times 10^{6}$ cells. The samples were incubated for $10 \mathrm{~min}$ at $37{ }^{\circ} \mathrm{C}$ under agitation. Then, a 5 $727 \mathrm{mM}$ glucose solution was added and incubated for 0 and $25 \mathrm{~min}$ at $37^{\circ} \mathrm{C}$ under agitation; at 728 the end of the incubation period, the cells were centrifuged, and the supernatants were 729 carefully taken and stored at $-70{ }^{\circ} \mathrm{C}$ for the further determination of L-lactate levels. 730 Standard values were determined from a $20 \mathrm{mM}$ L-lactate stock solution in distilled water 731 (standard curve: 0 to $500 \mu \mathrm{M}$ ). To generate the standard curve, aliquots of lactate were 732 added to a cuvette that contained $0.4 \mathrm{M}$ hydrazine, $0.5 \mathrm{M}$ glycine (pH 9.5), $1 \mathrm{mM} \mathrm{NAD}$, 733 and $20 \mathrm{U} / \mathrm{mL}$ L-lactate dehydrogenase at $25^{\circ} \mathrm{C}$. The reduction of $\mathrm{NAD}^{+}$was 734 spectrophotometrically (Cary $100 \mathrm{UV} / \mathrm{Vis}$ ) recorded at $340 \mathrm{~nm}$. To calculate the 735 extracellular lactate level in the samples, $50 \mu \mathrm{L}$ of supernatant was added to the cuvettes, 736 and $\mathrm{NAD}^{+}$reduction was recorded. Finally, the concentration of lactate was calculated 737 using the extinction coefficient for NADH $\left(\varepsilon=6,200 \mathrm{M}^{-1} \mathrm{~cm}^{-1}\right)$ and a standard curve. The 738 results are shown as the mean of four independent experiments and expressed as nmol of L739 lactate $/ \mathrm{h} / 1 \times 10^{6}$ cells. 


\section{MGO quantification.}

742 Intracellular free MGO was spectrophotometrically measured by using 2,4-

743 dinitrophenylhydrazine (DNPH) according to the method of Gilbert and Brandt [61] with

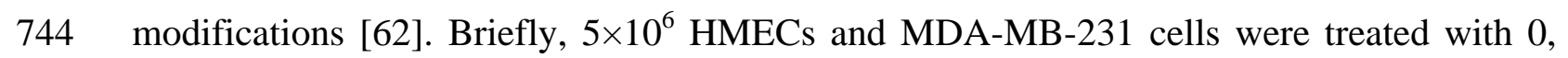
745700 , and $1000 \mu \mathrm{M}$ rabeprazole and 0,70 , and $150 \mu \mathrm{M}$ auranofin for $24 \mathrm{~h}$ under the 746 abovementioned general culture conditions. Cells were resuspended in PBS and lysed with 747 five freeze/thaw cycles, after which $0.45 \mathrm{M}$ perchloric acid was added to each sample, 748 chilled on ice for $10 \mathrm{~min}$, and centrifuged at $12,000 \mathrm{rpm}$ and $4{ }^{\circ} \mathrm{C}$ for $10 \mathrm{~min}$. The 749 supernatant was collected and stored at $-70^{\circ} \mathrm{C}$ for further measurement. Before determining 750 the MGO concentration in the samples, standard MGO values were calculated. Stock 751 solutions of $20 \mathrm{mM}$ DNPH in HCl-ethanol (12:88) and 0.1 mM MGO in distilled water 752 were prepared. Increasing concentrations of MGO (0 to $10 \mu \mathrm{M})$ were incubated with 0.2 $753 \mathrm{mM} \mathrm{DNPH}$ at $42{ }^{\circ} \mathrm{C}$ for $45 \mathrm{~min}$; then, the samples were cooled for $5 \mathrm{~min}$ at room 754 temperature, and the absorbance of MGO-bis-2,4-dinitrophenylhydrazone was recorded at $755432 \mathrm{~nm}$ on a microplate spectrophotometer (Epoch, BioTek, Winooski, VT, USA). Finally, 756 the cell supernatants were taken and used to quantify MGO levels with DNPH. The MGO 757 concentrations from the cells and standard curve were estimated using the extinction 758 coefficient $\varepsilon=33,600 \mathrm{M}^{-1} \mathrm{~cm}^{-1}$ for MGO-bis-2,4-dinitrophenyl-hydrazone and the 759 standard curve. The assays were carried out in triplicate to guarantee reproducibility. The 760 results are expressed as $[\mu \mathrm{M}] \mathrm{MGO} / 1 \times 10^{6}$ cells.

762 AGE quantification.

763 AGEs were determined by using an AGE ELISA kit with the manufacturer's instructions 764 (MyBioSource, San Diego, CA, USA). HMECs and MDA-MB-231 cells $\left(5 \times 10^{6}\right)$ were 765 treated with 0,700 , and $1000 \mu \mathrm{M}$ rabeprazole and 0,70 , and $150 \mu \mathrm{M}$ auranofin for $24 \mathrm{~h}$ 766 under the abovementioned culture conditions. After washing, cells were lysed with RIPA 767 buffer containing protease inhibitors, and the protein extract was separated. The protein 768 concentration was determined with the Bradford method.

769 The protein concentration in the samples was adjusted to $1 \mathrm{mg} / \mathrm{mL}$, after which the samples 770 were diluted 1:100 and loaded on ELISA plates to determine the AGE concentration. Then,

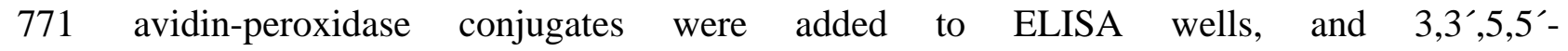


772 tetramethylbenzidine (TMB) was used as the substrate for coloring after the reactant was

773 thoroughly washed out with PBS. Additionally, a standard curve was made with the AGE

774 standard included in the kit. Standard concentrations were 0, 3.12, 6.25, 12.5, 25, 50, 100

775 and $200 \mathrm{ng} / \mathrm{mL}$. The absorbance at $450 \mathrm{~nm}$ was measured within the first $10 \mathrm{~min}$ in an

776 Epoch microplate spectrophotometer (BioTek, Vermont, USA). The results are the mean of

777 four independent experiments and are expressed as $\mu \mathrm{g}$ of AGEs/mL.

\section{Determining the factors related to induced cell death.}

780 To assess whether the induced cell death in breast cancer cells was due to apoptosis, assays 781 were conducted as follows. MDA-MB-231 cells $\left(1 \times 10^{7}\right)$ were treated with $0,500,1000$ and $7821500 \mu \mathrm{M}$ rabeprazole and $0,10,25$ and $50 \mu \mathrm{M}$ auranofin for $24 \mathrm{~h}$. Then, the cells were 783 resuspended in PBS and lysed in ice-cold RIPA buffer with protease inhibitors, and the 784 lysates were stored at $-70{ }^{\circ} \mathrm{C}$ until use. Protein samples were loaded into $16 \%$ SDS-PAGE 785 gels and transferred to PVDF membranes $\left(0.8 \mathrm{~mA} / \mathrm{cm}^{2}, 2 \mathrm{~h}\right)$ in $25 \mathrm{mM}$ Tris buffer, $192 \mathrm{mM}$ 786 glycine and 20\% methanol. After this, nonspecific binding sites were blocked with TBS-T 787 and 5\% BSA, and the membranes were washed one time with TBS-T and incubated 788 overnight at $4{ }^{\circ} \mathrm{C}$ with primary antibodies directed against ERK 1/2 (C-9), p-ERK1/2 789 (12D4), Caspase-7 (10-1-62), Bcl-2 (C-2), Bax (B-9) and $\beta$-Actin (C-2) (Santa Cruz 790 Biotechnology, Santa Cruz, CA, USA) at a dilution of 1:1000 in 0.1\% TBS-Tween-20 and 791 1\% BSA and washed three times with the same buffer. Proteins were immunoprobed using 792 an HRP-conjugated secondary antibody (dilution 1:3000) and chemiluminescent substrate 793 (Clarity Western ECL substrate, Bio-Rad, Hercules, CA, USA). Blot image acquisition was 794 performed using a Molecular Imager ${ }^{\circledR}$ Gel Doc ${ }^{\mathrm{TM}}$ XR+ system (Bio-Rad, CA, USA). The 795 optical density of the protein bands was calculated after background subtraction and normalization to $\beta$-Actin using Image Studio 4.0 software (LI-COR Biotechnology).

\section{TUNEL assays.}

799 To further confirm the induced apoptosis, the TUNEL method was employed with an In 800 situ Cell Death Detection Kit, Fluorescein 11684795910 (Roche, USA). Briefly, 5×10 801 MDA-MB-231 cells were grown over glass coverslips into six-well cell culture clusters 802 (Costar, USA) and incubated in the absence or presence of $700 \mu \mathrm{M}$ rabeprazole or $70 \mu \mathrm{M}$ 
803 auranofin for $24 \mathrm{~h}$ at $37{ }^{\circ} \mathrm{C}$. Then, the cells were washed three times with PBS and

804 incubated with Hanks balanced salt solution for $10 \mathrm{~min}$ at room temperature. Cells were

805 fixed with $100 \%$ methanol at $-20{ }^{\circ} \mathrm{C}$ for $15 \mathrm{~min}$; finally, the methanol was removed, and the

806 cells were rehydrated by washing with PBS 2 times for $5 \mathrm{~min}$ each. Samples were stained

807 with $1 \mathrm{mg} / \mathrm{mL}$ 4',6-diamidino-2-phenylindole (DAPI), washed with PBS and incubated

808 with proteinase $\mathrm{K}(20 \mathrm{mg} / \mathrm{mL})$ for $30 \mathrm{~min}$ at room temperature. Then, the cells were

809 washed with PBS and incubated for $30 \mathrm{~min}$ at room temperature with $0.3 \% \mathrm{H}_{2} \mathrm{O}_{2}$ in

810 methanol. Immediately after incubation with permeabilization solution $(0.1 \%$ Triton $\mathrm{X}-100$

811 in PBS) for $1 \mathrm{~h}$ at $4{ }^{\circ} \mathrm{C}$, the cells were washed three times with PBS and incubated in the

812 dark for $1 \mathrm{~h}$ at $37{ }^{\circ} \mathrm{C}$ with solutions $\mathrm{A}$ and B. Analysis was performed by fluorescence

813 microscopy (Axiovert, Carl Zeiss. Germany).

\section{Caspase-1 assays.}

815 Because HsTPI accumulated in breast cancer cells but not in HMECs, we hypothesized that

816 this condition is important to generate a favorable response to drug treatments. Thus, we

817 performed assays to mimic HsTPI accumulation in normal cells by inhibiting caspase-1 as

818 follows.

819 A stock solution (50 mM) of the selective caspase-1 inhibitor VX-765 [63], from BioVision

820 (Milpitas, CA) was prepared in DMSO and diluted in HMEC medium to the appropriate 821 concentrations. HMECs $\left(5 \times 10^{6}\right)$ were incubated for $24 \mathrm{~h}$ as previously mentioned with or 822 without $500 \mu \mathrm{M}$ caspase-1 inhibitor. Then, 0.5 and $1.5 \mathrm{mM}$ auranofin and rabeprazole, 823 respectively, were added to the samples and incubated for another $24 \mathrm{~h}$ under the same 824 conditions. At the end of incubation time, the cells were detached, washed and prepared for 825 the following assays as previously described: nPAGE, cell viability assay, HsTPI activity 826 assay, AGE quantification, assays to assess factors related to the induced cell death, and 827 TUNEL assays. To corroborate the inhibition of caspase-1, caspase-1 activity was 828 measured. In a buffer containing 100 mM HEPES (pH 7.2), $100 \mathrm{mM} \mathrm{NaCl}, 0.2 \%$ CHAPS, $82920 \mathrm{mM}$ EDTA, $10 \%$ glycerol and $10 \mathrm{mM}$ DTT, $500 \mu \mathrm{g}$ of total extract of cellular proteins, 830 the reaction was initiated in a 96-well plate (total reaction volume was $100 \mu \mathrm{L} /$ well) by the 831 addition of $200 \mu \mathrm{M}$ Ac-YVAD-pNA substrate.

832 The plate was then incubated for $4 \mathrm{~h}$ at $37{ }^{\circ} \mathrm{C}$, and at the end of the incubation period, the 833 absorbance at $405 \mathrm{~nm}$ was read using a microplate ELISA reader. 
834 This assay is based on the ability of the active enzyme to cleave the chromophore pNA, as

835 measured through determining the absorbance at $405 \mathrm{~nm}$.

836

837

838

839

840

841

842

843

844

845

861 The experiment finished $24 \mathrm{~h}$ after the last drug application in each group, after which the 862 animals were killed in $\mathrm{CO}_{2}$ chamber. Tumors (when they were present) were dissected 863 and prepared to analyze HsTPI as mentioned above.

\section{In vivo experiments.}

Five- to seven-week-old female athymic Balb/c nude mice $(\mathrm{Nu} / \mathrm{Nu})$ were obtained from the Círculo ADN S.A. de C.V. and housed in the Unidad de Investigación Preclínica of the National Autonomous University of Mexico under specific pathogen-free (SPF) conditions with free access to autoclaved food and water; the mice were cared for following NIH guidelines for laboratory animals. For studies of the presence and effect of deamidated HsTPI in tumors, the group sizes were chosen based on previous experience, and mice were randomly assigned to one of the following experimental groups: nontreatment $(n=4)$, subcutaneously injected once with $20 \times 10^{6}$ MDA-MB-231 cells and intraperitoneally administered sterile PBS three times a week; treated $(n=6)$, subcutaneously injected once with $20 \times 10^{6}$ MDA-MB-231 cells and intraperitoneally administered $50 \mathrm{mg} / \mathrm{kg}$ rabeprazole three times a week; pretreated $(n=2)$, subcutaneously injected once with $20 \times 10^{6}$ MDA-MB231 cells previously incubated with $1 \mathrm{mM}$ rabeprazole for $24 \mathrm{~h}$. The viability of the cells was verified before injection, and the injection volume was adjusted to guarantee that at least $20 \times 10^{6}$ of the injected cells were alive. Human cancer cells were implanted in mice by subcutaneous injection in the dorsal flank. The number of animals and all procedures followed protocols approved by the Institutional Animal Care and Use Committee for Care and Use (IACCUAC protocol UNIPREC-19-020).

Rabeprazole was intraperitoneal applied 7 days after the implantation of MDA-MB-231 cells. The animals were weighed, and the tumor length and width were measured using a digital caliper three times a week for 34 days. Using an established formula $(0.52 \mathrm{x}$ (length of the 'long axis' of the tumor) $\times$ (length of the 'short axis' of the tumor) ${ }^{2}$, tumor sizes were converted into tumor volumes [64].

864 Acknowledgments 
865 We thank to Bruno López-Fuentes for critical revision of the language. Daniela Kayani

866 Rangel-López designed Figure 6C. This research was funded by the Recursos Fiscales para

867 Investigación Program from the Instituto Nacional de Pediatría, S.S. Grant Number

$868 \quad 2019 / 072$.

\section{$871 \quad$ References}

1. Yin C, Qie S, Sang N. Carbon source metabolism and its regulation in cancer cells. Critical Reviews ${ }^{\mathrm{TM}}$ in Eukaryotic Gene Expression. 2012;22(1).

2. Keibler MA, Wasylenko TM, Kelleher JK, Iliopoulos O, Vander Heiden MG, Stephanopoulos G. Metabolic requirements for cancer cell proliferation. Cancer \&

$8783 . \quad$ Madhukar NS, Warmoes MO, Locasale JW. Organization of enzyme concentration across the metabolic network in cancer cells. PloS one. 2015;10(1):e0117131.

8804 4. Lincet H, Icard P. How do glycolytic enzymes favour cancer cell proliferation by 881 nonmetabolic functions? Oncogene. 2015;34(29):3751-9. Epub 2014/09/30. doi:

882 10.1038/onc.2014.320. PubMed PMID: 25263450.

883 5. Song S, Jacobson KN, McDermott KM, Reddy SP, Cress AE, Tang H, et al. ATP 884 promotes cell survival via regulation of cytosolic $[\mathrm{Ca}+]$ and $\mathrm{Bcl}-2 / \mathrm{Bax}$ ratio in lung cancer cells. American Journal of Physiology-Cell Physiology. 2016;310(2):C99-C114.

886 6. Zeng X, Liu L, Zheng M, Sun H, Xiao J, Lu T, et al. Pantoprazole, an FDAapproved proton-pump inhibitor, suppresses colorectal cancer growth by targeting T-cell-

889 7. Clem B, Telang S, Clem A, Yalcin A, Meier J, Simmons A, et al. Small-molecule 890 inhibition of 6-phosphofructo-2-kinase activity suppresses glycolytic flux and tumor 891 growth. Molecular cancer therapeutics. 2008;7(1):110-20.

892 8. Ganapathy-Kanniappan S, Geschwind J-FH. Tumor glycolysis as a target for cancer 893 therapy: progress and prospects. Molecular cancer. 2013;12(1):1-11.

8949 9. Pelicano H, Martin D, Xu R, and, Huang P. Glycolysis inhibition for anticancer 895 treatment. Oncogene. 2006;25(34):4633-46.

896 10. Enríquez-Flores S, Flores-López LA, García-Torres I, de la Mora-de la Mora I, 897 Cabrera N, Gutiérrez-Castrellón P, et al. Deamidated Human Triosephosphate Isomerase is 898 a Promising Druggable Target. Biomolecules. 2020;10(7). Epub 2020/07/19. doi:

899 10.3390/biom10071050. PubMed PMID: 32679775; PubMed Central PMCID:

900 PMCPMC7407242.

901 11. Guseman AJ, Whitley MJ, González JJ, Rathi N, Ambarian M, Gronenborn AM. 902 Assessing the Structures and Interactions of $\gamma \mathrm{D}-\mathrm{Crystallin}$ Deamidation Variants. Structure. 9032020.

904 12. de la Mora-de la Mora IT-L, Alfredo, Enríquez-Flores S, Méndez S-T, Castillo905 Villanueva A, Gómez-Manzo S, López-Velázquez G, et al. Structural effects of protein 906 aging: terminal marking by deamidation in human triosephosphate isomerase. Plos one. 907 2015;10(4):e0123379. 
908 13. Takata T, Oxford JT, Demeler B, Lampi KJ. Deamidation destabilizes and triggers

909 aggregation of a lens protein, $\beta$ A3-crystallin. Protein science. 2008;17(9):1565-75.

910 14. Gracy RW, Talent JM, Zvaigzne AI. Molecular wear and tear leads to terminal

911 marking and the unstable isoforms of aging. Journal of Experimental Zoology Part A:

912 Ecological Genetics and Physiology. 1998;282(1-2):18-27.

913 15. Decker R, Mohrenweiser HW. Cell proliferation-associated expression of a recently

914 evolved isozyme of triosephosphate isomerase. Biochemical genetics. 1985;23(3-4):267-80.

915 16. Harbut MB, Vilchèze C, Luo X, Hensler ME, Guo H, Yang B, et al. Auranofin

916 exerts broad-spectrum bactericidal activities by targeting thiol-redox homeostasis. Proc

917 Natl Acad Sci U S A. 2015;112(14):4453-8. Epub 2015/04/02. doi:

918 10.1073/pnas.1504022112. PubMed PMID: 25831516; PubMed Central PMCID:

919 PMCPMC4394260.

920 17. Shin JM, Kim N. Pharmacokinetics and pharmacodynamics of the proton pump

921 inhibitors. J Neurogastroenterol Motil. 2013;19(1):25-35. Epub 2013/01/26. doi:

922 10.5056/jnm.2013.19.1.25. PubMed PMID: 23350044; PubMed Central PMCID:

923 PMCPMC3548122.

924 18. Bornstein P, Balian G. Cleavage at Asn-Gly bonds with hydroxylamine. Methods

925 Enzymol. 1977;47:132-45. Epub 1977/01/01. doi: 10.1016/0076-6879(77)47016-2.

926 PubMed PMID: 927171.

927 19. Simpson RJ. Cleavage of asn-gly bonds by hydroxylamine. CSH Protoc.

928 2007;2007:pdb.prot4697. Epub 2007/01/01. doi: 10.1101/pdb.prot4697. PubMed PMID:

92921357038.

930 20. Kwong MY, Harris RJ. Identification of succinimide sites in proteins by N-terminal

931 sequence analysis after alkaline hydroxylamine cleavage. Protein Sci. 1994;3(1):147-9.

932 Epub 1994/01/01. doi: 10.1002/pro.5560030119. PubMed PMID: 8142891; PubMed

933 Central PMCID: PMCPMC2142483.

934 21. Gracy RW. Triosephosphate isomerase from human erythrocytes. Methods

935 Enzymol. 1975;41:442-7. Epub 1975/01/01. doi: 10.1016/s0076-6879(75)41096-5.

936 PubMed PMID: 1128275.

937 22. Leoncini G, Maresca M, Buzzi E. Inhibition of the glycolytic pathway by

938 methylglyoxal in human platelets. Cell Biochem Funct. 1989;7(1):65-70. Epub 1989/01/01.

939 doi: 10.1002/cbf.290070111. PubMed PMID: 2752537.

940 23. Ahmed N, Battah S, Karachalias N, Babaei-Jadidi R, Horányi M, Baróti K, et al.

941 Increased formation of methylglyoxal and protein glycation, oxidation and nitrosation in

942 triosephosphate isomerase deficiency. Biochim Biophys Acta. 2003;1639(2):121-32. Epub

943 2003/10/16. doi: 10.1016/j.bbadis.2003.08.002. PubMed PMID: 14559119.

944 24. Lowe SW, Lin AW. Apoptosis in cancer. Carcinogenesis. 2000;21(3):485-95. Epub

945 2000/02/26. doi: 10.1093/carcin/21.3.485. PubMed PMID: 10688869.

946 25. Lee JH, Parveen A, Do MH, Kang MC, Yumnam S, Kim SY. Molecular

947 mechanisms of methylglyoxal-induced aortic endothelial dysfunction in human vascular

948 endothelial cells. Cell Death \& Disease. 2020;11(5):1-15.

949 26. Mahali S, Raviprakash N, Raghavendra PB, Manna SK. Advanced glycation end

950 products (AGEs) induce apoptosis via a novel pathway: involvement of Ca2+ mediated by

951 interleukin-8 protein. J Biol Chem. 2011;286(40):34903-13. Epub 2011/08/25. doi:

952 10.1074/jbc.M111.279190. PubMed PMID: 21862577; PubMed Central PMCID:

953 PMCPMC3186367. 
954

955

956

957

958

959

960

961

962

963

964

965

966

967

968

969

970

971

972

973

974

975

976

977

978

979

980

981

982

983

984

985

986

987

988

989

990

991

992

993

994

995

996

997

998

999

27. Fink SL, Cookson BT. Apoptosis, pyroptosis, and necrosis: mechanistic description of dead and dying eukaryotic cells. Infect Immun. 2005;73(4):1907-16. Epub 2005/03/24. doi: 10.1128/iai.73.4.1907-1916.2005. PubMed PMID: 15784530; PubMed Central PMCID: PMCPMC1087413.

28. Hsu SY, Hsueh AJ. A splicing variant of the Bcl-2 member Bok with a truncated BH3 domain induces apoptosis but does not dimerize with antiapoptotic Bcl-2 proteins in vitro. J Biol Chem. 1998;273(46):30139-46. Epub 1998/11/07. doi: 10.1074/jbc.273.46.30139. PubMed PMID: 9804769.

29. Willis S, Day CL, Hinds MG, Huang DC. The Bcl-2-regulated apoptotic pathway. J Cell Sci. 2003;116(Pt 20):4053-6. Epub 2003/09/16. doi: 10.1242/jcs.00754. PubMed PMID: 12972498.

30. Shao W, Yeretssian G, Doiron K, Hussain SN, Saleh M. The caspase-1 digestome identifies the glycolysis pathway as a target during infection and septic shock. J Biol Chem. 2007;282(50):36321-9. Epub 2007/10/26. doi: 10.1074/jbc.M708182200. PubMed PMID: 17959595 .

31. Kumar S, van Raam BJ, Salvesen GS, Cieplak P. Caspase cleavage sites in the human proteome: CaspDB, a database of predicted substrates. PLoS One.

2014;9(10):e110539. Epub 2014/10/21. doi: 10.1371/journal.pone.0110539. PubMed PMID: 25330111; PubMed Central PMCID: PMCPMC4201543.

32. Rawlings ND, Waller M, Barrett AJ, Bateman A. MEROPS: the database of proteolytic enzymes, their substrates and inhibitors. Nucleic Acids Res. 2014;42(Database issue):D503-9. Epub 2013/10/26. doi: 10.1093/nar/gkt953. PubMed PMID: 24157837; PubMed Central PMCID: PMCPMC3964991.

33. Sun Y, Guo Y. Expression of Caspase-1 in breast cancer tissues and its effects on cell proliferation, apoptosis and invasion. Oncol Lett. 2018;15(5):6431-5. Epub 2018/05/05. doi: 10.3892/ol.2018.8176. PubMed PMID: 29725399; PubMed Central PMCID: PMCPMC5920210.

34. Otto T, Sicinski P. Cell cycle proteins as promising targets in cancer therapy. Nat Rev Cancer. 2017;17(2):93-115. Epub 2017/01/28. doi: 10.1038/nrc.2016.138. PubMed PMID: 28127048; PubMed Central PMCID: PMCPMC5345933.

35. Visweswaran M, Arfuso F, Warrier S, Dharmarajan A. Aberrant lipid metabolism as an emerging therapeutic strategy to target cancer stem cells. Stem Cells. 2020;38(1):6-14. Epub 2019/10/28. doi: 10.1002/stem.3101. PubMed PMID: 31648395.

36. Thongwatchara P, Promwikorn W, Srisomsap C, Chokchaichamnankit D, Boonyaphiphat $\mathrm{P}$, Thongsuksai P. Differential protein expression in primary breast cancer and matched axillary node metastasis. Oncol Rep. 2011;26(1):185-91. Epub 2011/04/20. doi: 10.3892/or.2011.1266. PubMed PMID: 21503584.

37. Pekel G, Ari F. Therapeutic Targeting of Cancer Metabolism with Triosephosphate Isomerase. Chem Biodivers. 2020;17(5):e2000012. Epub 2020/03/18. doi: 10.1002/cbdv.202000012. PubMed PMID: 32180338.

38. Waks AG, Winer EP. Breast Cancer Treatment: A Review. Jama. 2019;321(3):288300. Epub 2019/01/23. doi: 10.1001/jama.2018.19323. PubMed PMID: 30667505.

39. Skinner K, Dufour R, Haiderali A, Huang M, Schwartzberg L. PCN131ASSESSING THE REAL-WORLD COST OF CARE IN PATIENTS WITH METASTATIC TRIPLE NEGATIVE BREAST CANCER (MTNBC) IN THE UNITED STATES. Value in Health. 2018;21:S36. 
1000 40. Allaman I, Bélanger M, Magistretti PJ. Methylglyoxal, the dark side of glycolysis.

1001

1002

1003

1004

1005

1006

1007

1008

1009

1010

1011

1012

1013

1014

1015

1016

1017

1018

1019

1020

1021

1022

1023

1024

1025

1026

1027

1028

1029

1030

1031

1032

1033

1034

1035

1036

1037

1038

1039

1040

1041

1042

1043

1044

1045

1046

Front Neurosci. 2015;9:23. Epub 2015/02/25. doi: 10.3389/fnins.2015.00023. PubMed PMID: 25709564; PubMed Central PMCID: PMCPMC4321437.

41. Orosz F, Oláh J, Ovádi J. Triosephosphate isomerase deficiency: new insights into an enigmatic disease. Biochim Biophys Acta. 2009;1792(12):1168-74. Epub 2009/09/30. doi: 10.1016/j.bbadis.2009.09.012. PubMed PMID: 19786097.

42. Okado A, Kawasaki Y, Hasuike Y, Takahashi M, Teshima T, Fujii J, et al. Induction of apoptotic cell death by methylglyoxal and 3-deoxyglucosone in macrophagederived cell lines. Biochem Biophys Res Commun. 1996;225(1):219-24. Epub 1996/08/05. doi: 10.1006/bbrc.1996.1157. PubMed PMID: 8769121.

43. Ott C, Jacobs K, Haucke E, Navarrete Santos A, Grune T, Simm A. Role of advanced glycation end products in cellular signaling. Redox Biol. 2014;2:411-29. Epub 2014/03/14. doi: 10.1016/j.redox.2013.12.016. PubMed PMID: 24624331; PubMed Central PMCID: PMCPMC3949097.

44. van Marion DM, Domanska UM, Timmer-Bosscha H, Walenkamp AM. Studying cancer metastasis: Existing models, challenges and future perspectives. Critical reviews in oncology/hematology. 2016;97:107-17.

45. Zhang S, Wang Y, Li SJ. Lansoprazole induces apoptosis of breast cancer cells through inhibition of intracellular proton extrusion. Biochemical and biophysical research communications. 2014;448(4):424-9.

46. Kold-Christensen R, Johannsen M. Methylglyoxal Metabolism and Aging-Related Disease: Moving from Correlation toward Causation. Trends Endocrinol Metab.

2020;31(2):81-92. Epub 2019/11/24. doi: 10.1016/j.tem.2019.10.003. PubMed PMID: 31757593.

47. Nokin MJ, Durieux F, Bellier J, Peulen O, Uchida K, Spiegel DA, et al. Hormetic potential of methylglyoxal, a side-product of glycolysis, in switching tumours from growth to death. Sci Rep. 2017;7(1):11722. Epub 2017/09/17. doi: 10.1038/s41598-017-12119-7. PubMed PMID: 28916747; PubMed Central PMCID: PMCPMC5600983.

48. Pekel G, Ari F. Therapeutic Targeting of Cancer Metabolism with Triosephosphate Isomerase. Chemistry \& biodiversity. 2020;17(5):e2000012.

49. Li C, Xiao Z, Chen Z, Zhang X, Li J, Wu X, et al. Proteome analysis of human lung squamous carcinoma. Proteomics. 2006;6(2):547-58.

50. Cui J-W, Wang J, He K, Jin B-F, Wang H-X, Li W, et al. Proteomic analysis of human acute leukemia cells: insight into their classification. Clinical cancer research. 2004;10(20):6887-96.

51. Pettersen EF, Goddard TD, Huang CC, Couch GS, Greenblatt DM, Meng EC, et al. UCSF Chimera--a visualization system for exploratory research and analysis. J Comput Chem. 2004;25(13):1605-12. Epub 2004/07/21. doi: 10.1002/jcc.20084. PubMed PMID: 15264254.

52. Morris GM, Huey R, Lindstrom W, Sanner MF, Belew RK, Goodsell DS, et al. AutoDock4 and AutoDockTools4: Automated docking with selective receptor flexibility. J Comput Chem. 2009;30(16):2785-91. Epub 2009/04/29. doi: 10.1002/jcc.21256. PubMed PMID: 19399780; PubMed Central PMCID: PMCPMC2760638.

53. Trott O, Olson AJ. AutoDock Vina: improving the speed and accuracy of docking with a new scoring function, efficient optimization, and multithreading. J Comput Chem. 2010;31(2):455-61. Epub 2009/06/06. doi: 10.1002/jcc.21334. PubMed PMID: 19499576; PubMed Central PMCID: PMCPMC3041641. 
1047

1048

1049

1050

1051

1052

1053

1054

1055

1056

1057

1058

1059

1060

1061

1062

1063

1064

1065

1066

1067

1068

1069

1070

1071

1072

1073

1074

1075

1076

1077

1078

1079

1080

1081

1082

1083

1084

1085

54. Grimsley GR, Pace CN. Spectrophotometric determination of protein concentration. Curr Protoc Protein Sci. 2004;Chapter 3:Unit 3.1. Epub 2008/04/23. doi: 10.1002/0471140864.ps0301s33. PubMed PMID: 18429266.

55. García-Torres I, de la Mora-de la Mora I, Marcial-Quino J, Gómez-Manzo S, Vanoye-Carlo A, Navarrete-Vázquez G, et al. Proton pump inhibitors drastically modify triosephosphate isomerase from Giardia lamblia at functional and structural levels, providing molecular leads in the design of new antigiardiasic drugs. Biochim Biophys Acta. 2016;1860(1 Pt A):97-107. Epub 2015/11/01. doi: 10.1016/j.bbagen.2015.10.021. PubMed PMID: 26518348.

56. Plaut B, Knowles JR. pH-dependence of the triose phosphate isomerase reaction. Biochem J. 1972;129(2):311-20. Epub 1972/09/01. doi: 10.1042/bj1290311. PubMed PMID: 4643319; PubMed Central PMCID: PMCPMC1174081.

57. Ellman GL. A colorimetric method for determining low concentrations of mercaptans. Arch Biochem Biophys. 1958;74(2):443-50. Epub 1958/04/01. doi: 10.1016/0003-9861(58)90014-6. PubMed PMID: 13534673.

58. McLellan T. Electrophoresis buffers for polyacrylamide gels at various $\mathrm{pH}$. Anal Biochem. 1982;126(1):94-9. Epub 1982/10/01. doi: 10.1016/0003-2697(82)90113-0. PubMed PMID: 7181120.

59. Loveland BE, Johns TG, Mackay IR, Vaillant F, Wang ZX, Hertzog PJ. Validation of the MTT dye assay for enumeration of cells in proliferative and antiproliferative assays. Biochem Int. 1992;27(3):501-10. Epub 1992/07/01. PubMed PMID: 1417886.

60. Bergmeyer H, Bergmeyer J, Grassl M. Methods of enzymatic analysis vol. VI, 3rd edn. Verlag Chemie. Weinheim; 1983.

61. Gilbert RP, Brandt RB. Spectrophotometric determination of methyl glyoxal with 2, 4-dinitrophenylhydrazine. Analytical Chemistry. 1975;47(14):2418-22.

62. López-Velázquez G, Fernández-Lainez C, de la Mora-de JI, de la Portilla DC,

Reynoso-Robles R, González-Maciel A, et al. On the molecular and cellular effects of omeprazole to further support its effectiveness as an antigiardial drug. Scientific reports. 2019;9(1):1-14.

63. Boxer MB, Quinn AM, Shen M, Jadhav A, Leister W, Simeonov A, et al. A highly potent and selective caspase 1 inhibitor that utilizes a key 3-cyanopropanoic acid moiety. ChemMedChem. 2010;5(5):730-8.

64. Lee E, Lee SJ, Koskimaki JE, Han Z, Pandey NB, Popel AS. Inhibition of breast cancer growth and metastasis by a biomimetic peptide. Sci Rep. 2014;4:7139. Epub 2014/11/21. doi: 10.1038/srep07139. PubMed PMID: 25409905; PubMed Central PMCID: PMCPMC4238022 of AsclepiX Therapeutics, LLC; the terms of this arrangement are being managed by the Johns Hopkins University in accordance with its conflict of interest policies. 